\title{
Medievalista
}

Online

$28 \mid 2020$

Número 28

\section{Les évêques de Provence et la diplomatie royale sous Charles II (1285-1309)}

The Bishops of Provence and royal diplomacy under Charles II, 1285-1309

\section{Thierry Pécout}

\section{(2) OpenEdition}

1 Journals

Édition électronique

URL : http://journals.openedition.org/medievalista/3323

DOI : 10.4000/medievalista.3323

ISSN : 1646-740X

Éditeur

Instituto de Estudos Medievais - FCSH-UNL

\section{Édition imprimée}

Pagination : Bishop, Provence, Diplomacy, Provost, Dynasty of Provence-Sicily

\section{Référence électronique}

Thierry Pécout, « Les évêques de Provence et la diplomatie royale sous Charles II (1285-1309) », Medievalista [En ligne], 28 | 2020, mis en ligne le 01 juillet 2020, consulté le 23 mars 2021. URL : http:// journals.openedition.org/medievalista/3323 ; DOI : https://doi.org/10.4000/medievalista.3323

Ce document a été généré automatiquement le 23 mars 2021.

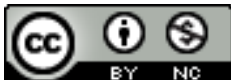

Mediavalista está licenciado com uma Licença Creative Commons - Atribuição-NãoComercial 4.0 Internacional. 


\title{
Les évêques de Provence et la diplomatie royale sous Charles II (1285-1309)
}

The Bishops of Provence and royal diplomacy under Charles II, 1285-1309

\author{
Thierry Pécout
}

\section{NOTE DE L'AUTEUR}

Registres des papes édités par l'École française de Rome: abréviations suivies du nom du pontife; $L$ : lettres ou registres, $L C$ : Lettres communes, $L C u$ : Lettres curiales. Pour alléger des notes déjà nourries, nous n'avons pas cité systématiquement ces mêmes lettres avec, quand le cas se présente, un renvoi à leur édition intégrale dans DOMÍNGUEZ SÁNCHEZ, Santiago (éd.) - Documentos de Martin IV (1281-1285) referentes a España. León: Universidad de León, 2010; DOMÍNGUEZ SÁNCHEZ, Santiago (éd.) Documentos de Honorio IV (1285-1287) referentes a España. León: Universidad de León, 2015; DOMÍNGUEZ SÁNCHEZ, Santiago (éd.) - Documentos de Nicolás IV (1288-1292) referentes a España. León: Universidad de León, 2009; DOMÍNGUEZ SÁNCHEZ, Santiago (éd.) Documentos de Bonifacio VIII (1294-1303) referentes a España. León: Universidad de León, 2006; DOMÍNGUEZ SÁNCHEZ, Santiago (éd.) - Documentos de Clemente V (1305-1314) referentes a España. León: Universidad de León, 2014.

1 Lors d'un colloque tenu à Szeged en 2007, j'avais étudié l'activité d'un procureur de la reine Jeanne de Naples in Romana Curia, au milieu du XIV siècle $^{1}$. À l'occasion de la présentation des actes de cette manifestation par l'Institutum Pontificium Ecclesiasticum Hungaricum in Urbe, je m'étais interrogé comme bien d'autres sur l'existence de la diplomatie aux temps médiévaux. Évidemment, nous entendions par là que la pratique diplomatique ne connaissait pas alors de spécialisation, ni en tant qu'art politique autonome, ni en tant que profession. La diplomatie et le diplomate sont intimement liés à l'émergence d'un droit des gens et de la notion juridique d'état de paix. Mais 
assurément aussi, la diplomatie en tant que nécessité politique de gestion des rapports de force et des rivalités entre puissances, quelle que soit leur échelle, n'a pas attendu les temps modernes pour émerger². Et c'est là justement la spécificité de la diplomatie médiévale, que de n'être avant tout que praxis et de s'intégrer dans une relation globale. Le "diplomate" de ces temps, est celui qui met à disposition un réseau personnel et professionnel, issu tant de sa parenté que des affinités créées au cours de ses études et de sa carrière; qui entretient avec le prince des relations personnelles, voire intime, puisqu'il en est la bouche et les yeux; qui s'avère expert dans le ministère de la parole, de la négociation et qui impose sa reconnaissance à ses interlocuteurs par certaine vertu politique, renommée ou charisme. À la lecture de cette énumération, on ne s'étonnera guère de voir ainsi se profiler avec elle un personnel issu du sacerdoce, de l'hôtel, de la ville et de l'Université. À propos des Angevins de Provence-Sicile, quelques carrières significatives nous permettront d'illustrer ces considérations, entre les années 1280 et les premiers temps de la papauté d'Avignon, moment où le cadre provençal s'impose comme haut-lieu de pourparlers et zone de contacts, dans un contexte hautement conflictuel en Méditerranée occidentale ${ }^{3}$. La première maison d'Anjou-Provence-Sicile régna en effet sur les comtés de Provence et de Forcalquier puis d'Anjou et du Maine à partir de 1246 et accéda à la couronne de Sicile en 1265 avec le soutien de la papauté. À partir de 1282, elle entra dans un cycle de conflits avec la couronne d'Aragon.

2 À compter du règne de Charles II (1285-1309), l'épiscopat de Provence semble se déterminer en fonction du rôle que lui assigne la monarchie dans son projet d'administration du peuple chrétien. En cela, il se rapproche de plus en plus du milieu des grands officiers. L'épiscopat poursuit un processus d'homogénéisation politique entamé au début du siècle ${ }^{4}$. Il s'achemine vers une communauté de pensée reposant sur une formation intellectuelle dominée par le droit, un même idéal de rationalisation du gouvernement chrétien, déployant particulièrement une fonction médiatrice qui s'investit dans la diplomatie ${ }^{5}$.

3 Deux types d'hommes d'Église caractérisent ce processus. Tout d'abord, des proches du souverain qui s'illustrent par une carrière bénéficiale prestigieuse, mais d'un rang auprès du prince bien supérieur à leur statut dans l'Église locale. Ils préfèrent des bénéfices sine cura, avec une ordination sacerdotale tardive, et n'accèdent pas à l'épiscopat, en partie par choix. Ils font un usage pragmatique du bénéfice ecclésiastique. Ainsi de Guillaume de Ferrières ( $† 1295)$ et de Guillaume Agarini ( $† 1311)$, qui demeurent prévôts pendant presque toute leur carrière, respectivement à Marseille et à Apt. Toutefois, le premier accède au cardinalat, tandis que l'autre échoue à l'épiscopat. Au service du roi, Guillaume Agarini a grandement concouru à structurer l'office de procureur in romana curia. À côté d'eux, viennent des prélats ponctuellement chargés de missions diplomatiques. Ce sont des auxiliaires du souverain, sans postes dans l'appareil administratif. Ainsi de Rostaing de Capra (1286-1303), Rostaing de Noves (1283-1311), archevêques d'Arles et d'Aix, et d'André d'Anguissola (1291-1294), évêque d'Avignon'.

Guillaume de Ferrières illustrera ici le premier cas de figure, car je n'insisterai pas sur Guillaume Agarini dont j'ai déjà présenté ailleurs l'action fondatrice comme procureur royal ${ }^{7}$. Guillaume de Ferrières est un universitaire et un homme de loi. Issu d'une famille toulousaine, il se forme à l'université d'Orléans. Professeur de droit civil, il enseigne à Toulouse entre 1284 et 1289: il en est le premier docteur dont l'œuvre soit 
connue. Quand il paraît aux côtés du roi, il dispose déjà d'une longue expérience savante, qui s'est exercée dans la consultation juridique et illustrée en un recueil sur la coutume, des leçons sur les Institutes de 1285-1286, des traités et gloses. Il est fiscaliste, spécialiste de droit municipal et de procédure, ainsi que des juridictions entre roi et seigneurs ${ }^{8}$. Il mène une belle carrière bénéficiale en Provence en œuvrant au service de Charles II. Il est pourvu de la prévôté de Marseille en mai 1289 par Nicolas IV, alors qu'il n'est que clerc tonsuré, après la nomination du catalan Hugo de Mataplana au siège de Saragosse ${ }^{9}$. C'est sa fonction auprès du prince qui explique sa promotion: dès 1290 , Guillaume porte la titulature de familier et conseiller, tout comme celles de clerc du roi et de chapelain de Nicolas IV. Du reste, il est nommé vice-chancelier du royaume de Sicile peu avant mai $1290^{10}$. Il entretient une grande proximité avec les milieux gouvernant alors la Provence. On le rencontre fréquemment aux côtés des grands officiers de la cour d'Aix entre 1291 et $1294^{11}$. Il est probablement présent à Perugia avec Charles II et son fils Charles Martel († 1295) lors du conclave en mars 129412. Son accession au cardinalat, comme prêtre de San Clemente le 18 septembre 1294 par la grâce de Célestin V, en fait l'un des chefs de file du parti angevin au Sacré Collège ${ }^{13}$. Il demeure bien en cour auprès de Boniface VIII aussi ${ }^{14}$. Il supervise les négociations de mariage entre Blanche d'Anjou ( $† 1310$ ), fille de Charles II, et Jacques II d'Aragon (1291-1327) qui vient de laisser la couronne de Sicile à son frère Frédéric ${ }^{15}$. C'est alors une période complexe des relations entre Angevins et Aragonais, entre état de guerre et négociations. Une paix se conclut le 12 juin 1295 à Agnani, avec comme dispositif la renonciation à toute union entre Sicile et Aragon, l'indépendance du royaume de Majorque, la création d'un royaume de Sardaigne et Corse pour Jacques, l'abandon par Philippe IV (1285-1314) de toute prétention sur l'Aragon, le mariage de Jacques et Blanche, dont la dot sera financée par le pape (le mariage est célébré le $1^{\mathrm{er}}$ novembre 1295), et la libération des fils de Charles $\mathrm{II}^{16}$. Mais Guillaume de Ferrières décède prématurément à Perpignan, le 7 septembre 1295. D'autres négociateurs prennent le relais, tandis que son neveu ou frère Pierre de Ferrières prolonge sa carrière par bien des aspects.

5 L'action diplomatique fut vraisemblablement le principal ressort de l'éphémère carrière de Guillaume de Ferrières. Pour certains de ses collègues elle ne joue qu'un rôle secondaire, à l'occasion de missions ponctuelles. Des liens étroits noués avec l'entourage pontifical ont sans doute incliné le souverain à les leur confier. Au vrai, les deux cas ici évoqués sont liés au Sacré Collège et plus particulièrement au cardinal de Porto Bernard d'Anguissola ( $† 1291$ ), archevêque d'Arles en 1274 sous Clément IV et nommé cardinal par Martin IV en 1281. Ils occupent des sièges où l'emprise royale est traditionnellement peu présente, au profit de celle du pape. Ce statut leur permet d'occuper des fonctions médiatrices dans les négociations des années 1290.

6 Rostaing de Capra (†22 août 1303) procède du chapitre cathédral d'Arles et vraisemblablement d'une famille locale. C'est un régulier, comme l'est ce chapitre. Il est chanoine et prêtre depuis 1278 , ouvrier et administrateur des anniversaires en $1284^{17}$. Le 5 août 1286, il est pourvu comme archevêque d'Arles, en pleine période de vacance du pouvoir royal, alors que Charles II est en captivité en Aragon ${ }^{18}$. Il est membre de la familia du cardinal Bernard d'Anguissola, son prélat consécrateur. Le pape l'affecte à des tâches concourant au sauvetage de la monarchie angevine. À la fin de son pontificat, Honorius IV (1285-1287) lui confie ainsi le soin de transférer la décime en faveur du roi de Sicile et derechef sous Nicolas IV (1288-1292) ${ }^{19}$. Après le retour du roi, Rostaing est attesté dans sa suite, ce qui est inédit pour un archevêque d'Arles. Il porte 
les titres de familier et conseiller en 1294, sans pour autant exercer de fonction dans l'appareil administratif angevin ${ }^{20}$. Il effectue un voyage diplomatique dans les Pyrénées avec Charles II, ponctué par l'échec des négociations de Guillaume de Villaret, prieur hospitalier de Saint-Gilles ${ }^{21}$. En septembre 1295, il se trouve à Perpignan avec Guillaume de Ferrières ${ }^{22}$. Il le remplace sur ordre de Boniface VIII ${ }^{23}$. L'année suivante, il est nonce du pape ${ }^{24}$. Il joue ainsi un rôle de médiation et d'information entre la curie et la cour, particulièrement au sujet des affaires de Sicile et d'Aragon.

列 bien plus limitée. Il est frère du cardinal de Porto. L'aide de l'aîné est déterminante pour la carrière du cadet ${ }^{25}$. En 1290, André accède au siège d'Avignon par la faveur du pape $^{26}$. Son épiscopat dans une cité frontalière le conduit à jouer un rôle diplomatique précis. Au début de l'année 1291, il participe ainsi aux négociations de Tarascon, associant Charles II, le légat et cardinal Gerardo Bianchi, Benedetto Caetani, Charles de Valois, Jacques de Majorque, des représentants de Philippe IV, d'Édouard I ${ }^{\text {er }}$ d'Angleterre (1272-1307), d'Alphonse III de Castille (1285-1291) et des Cortes d'Aragon ${ }^{27}$. Elles aboutissent au traité de Brignoles, du 19 février 129128. André d'Anguissola n'intervient alors qu'en tant qu'évêque d'Avignon, la coseigneurie de celle ville étant l'objet d'un échange entre Philippe IV et Charles II qui l'obtient moyennant la cession de l'Anjou et du Maine à Charles de Valois ${ }^{29}$. André est toutefois membre du conseil royal et familier en $1292^{30}$.

8 Sous Charles II, le clergé séculier et singulièrement l'épiscopat exercent une activité accrue dans le gouvernement de la monarchie, à la mesure de leur rôle déterminant dans la survie de celle-là durant la crise de 1282-1288, après les Vêpres siciliennes, la défaite et la captivité en Aragon du jeune souverain. Tandis que se déploie leur magistère et que se redéfinit l'officium episcopi au sein de la théocratie pontificale, leur fonction médiatrice s'investit dans l'activité diplomatique.

9 Mais le sacerdoce n'est point toujours le socle à partir duquel se déploie celle-ci. Pour certains de nos clercs, la prélature couronne à l'inverse ou accompagne une éminente carrière de grand officier. Elle s'appuie sur des stratégies bénéficiales différenciées. Dans tous les cas cependant, office et prélature trouvent à s'imbriquer car ils participent d'un même magistère de la parole. À chaque fois aussi, un milieu s'esquisse dont les réseaux mettent en jeu la nécessité désormais de disposer de solides capacités de recueil de l'information auprès du gouvernement central de l'Église.

Voici en premier lieu deux distingués ministres, entre Église et État pour paraphraser Bernard Guenée ${ }^{31}$. Pierre de Ferrières et Guillaume de Mandagout se caractérisent par une indéniable stature intellectuelle, l'un dans le droit civil, l'autre dans le droit canon. Ils œuvrent chacun au service de deux appareils d'État, le premier pour le roi, le second pour le pape. L'obtention de l'archiépiscopat marque leur cursus bénéficial, dont l'un seulement aboutit au cardinalat, faute de longévité suffisante pour l'autre. La diplomatie y occupe une place singulière, parmi les activités qui distinguent ces deux hommes d'État.

Pierre de Ferrières († 1308), procède de la familia cardinalice de Guillaume, son parent ${ }^{32}$. Il est aussi familier de Louis d'Anjou, comme toute une génération ${ }^{33}$. Chez lui, le bénéfice rétribue l'office exercé auprès du souverain. Il préfigure par bien des aspects l'ascension de Jacques Duèze et sans doute Pierre aurait-il reçu le cardinalat s'il avait vécu davantage. Docteur en droit civil de l'université de Toulouse et gradué dans les deux droits, il y enseigne jusqu'en 1294. Il devient recteur du studium de Naples ${ }^{34}$. 
Trésorier, vice-chancelier du royaume de Sicile dès novembre 1295, avec le titre d'auditeur en 1298, c'est un grand officier ${ }^{35}$. Il est vicaire général dans le Regno en 1305 et promulgue des statuts sur les juridictions en Provence en 1304. Doté de la prêtrise en 1295, il cumule les bénéfices ${ }^{36}$ : doyen du Puy en 1295, puis de Douai, évêque de Lectoure en 1299, de Noyon en 1301, chanoine de Lyon et d'Auch, outre divers revenus dans les diocèses du Puy et de Viviers ${ }^{37}$. Il refuse cependant l'archevêché de Salerno en $1298^{38}$, mais accepte celui d'Arles en $1304^{39}$ : sa résidence en Provence semble essentielle à ses fonctions.

12 Il assure de nombreuses missions pour son maittre le roi. En Italie du nord tout d'abord, auprès de Manfredo marquis de Saluzzo en 1305 et de la commune de Gênes en $1307^{40}$. Puis auprès du nouveau pape Clément V, à Poitiers avec son maître Charles II en 1307. Il présente alors un mémoire sur les dettes de la monarchie, avec le protonotaire et logothète Bartolomeo di Capua, en arguant du rôle joué par celle-ci dans la défense de l'Église romaine ${ }^{41}$. Il combine la maîtrise de la parole et de l'art oratoire, le savoir juridique et la connaissance des milieux entourant le pontife. Comme la première génération de professeurs de droit civil entourant les Angevins en Provence, le droit semble pour lui continuer une entreprise de domination politique et militaire ${ }^{42}$. Mais la génération de Pierre de Ferrières ajoute une œuvre considérable de mise en ordre des juridictions et des instances dans les comtés provençaux.

13 De son côté, Guillaume de Mandagout ( $† 1321$ ) provient de la basse vallée du Rhône et du royaume de France ${ }^{43}$. Il débute dans le chapitre de Nîmes dès 1275. Il s'inscrit à l'origine dans ce modèle d'ascension bénéficiale dont le point de départ est la familia du cardinal Bernard d'Anguissola, mais il s'en distingue rapidement ${ }^{44}$. Par ses compétences intellectuelles, il dépasse des collègues tels Jacques Duèze, Bérenger Frédol et Pierre de Ferrières ${ }^{45}$. En outre, son cursus prolonge une stratégie de promotion lignagère mise en place par les siens dès les années 1260 et fondée sur le cumul bénéficial. Bien plus que pour Pierre de Ferrières étroitement lié au seul Charles II, sa fonction de médiation est remarquable, entre papauté, capétiens et Angevins et marque toute sa carrière, y compris lors de ses tentatives d'élection en conclave.

Guillaume de Mandagout est docteur du studium de Bologne et doit son ascension à son statut de savant juriste. Il est archidiacre d'Uzès, notaire apostolique en 1291, chapelain du cardinal Bernard d'Anguissola en 1286, prévôt de Toulouse en 129146. En avril 1295, Boniface VIII le pourvoit archevêque d'Embrun ${ }^{47}$. Parallèlement, il débute un travail de compilation juridique en 1296: le Sexte promulgué en 1298, les Clémentines probablement aussi, ainsi qu'un libelle sur les élections ${ }^{48}$. Clément $\mathrm{V}$ le promeut archevêque d'Aix en mai 1311, tandis que son chapelain lui succède à Embrun ${ }^{49}$. En décembre 1312 enfin, il devient évêque de Palestrina.

Son activité diplomatique est intense. Elle est favorisée par sa dispense de résidence dans ses bénéfices obtenue en $1297^{50}$. Mais dès 1291 il est dépêché auprès du roi d'Aragon et à Majorque ${ }^{51}$. Les questions touchant au royaume de Sicile occupent une place prépondérante. Guillaume de Mandagout est un intermédiaire précieux auprès du pape. Il participe lui aussi à la mission de 1295 à la suite de la mort de Guillaume de Ferrières, notamment lors des négociations finales de la fin de l'année 1295 et du début de la suivante, qui débouchent sur le mariage de Blanche d'Anjou et de Jacques d'Aragon en novembre $1295^{52}$. Il œuvre aussi à propos du Dauphiné, en particulier au temps de son épiscopat à Embrun. En juillet 1301, il mène les tractations entre le dauphin et Amédée V de Savoie, sous l'arbitrage de Charles de Valois et avec l'épiscopat 
local, ses collègues de Gap, Valence et Vienne ${ }^{53}$. En 1304, il est désigné par Clément V comme protecteur de Béatrice de Savoie Faucigny ${ }^{54}$. En 1300, il arbitre entre Charles II et le dauphin sur la question de Gap et de ses juridictions, aux côtés de Jacques Duèze ${ }^{55}$. Enfin, tout comme Bernard d'Anguissola à l'issue de son archiépiscopat à Arles, il est désigné comme recteur du comté Venaissin, où il est attesté entre 1303 et $1310^{56}$. En 1304, il prononce une consultation juridique sur l'héritage des Montauban, un lignage des confins dauphinois de la Provence, mais essuie les reproches de Charles II $^{57}$. En 1307, il joue sans doute un rôle déterminant dans le transfert de la moitié de la cité d'Orange au roi, ce qui occasionne un bouleversement des rapports de force aux marges du Venaissin pontifical ${ }^{58}$.

Son activité diplomatique est l'une des manifestations de la puissance et de l'étendue de ses réseaux personnels. Elle le conduit souvent à rechercher un difficile équilibre entre les puissances, dont il fait les frais en 1307 à propos des Montauban. Il ne s'agit pas, à la différence de Pierre de Ferrières, d'un agent angevin. Il dispose d'un réseau de relations qui tend à s'autonomiser et qu'il s'efforce de mettre au service de sa propre carrière bénéficiale.

17 Pour certains clercs, la fonction médiatrice et diplomatique passe enfin par des missions fiscales, qui structurent leurs carrières bénéficiales. La décime, arme politique par excellence dès ses régulières concessions au roi de Sicile, en est le principal acteur. La fonction de collecteur échoit souvent à des prévôts de chapitres cathédraux. Cette dignité comporte déjà des obligations de gestion temporelle et de reddition des comptes et c'est sans conteste une excellente école pour le futur comptable ${ }^{59}$. Ce bénéfice peut de surcroît être occupé par un titulaire absentéiste. La collecte de la décime, ou du moins sa supervision, est une activité qui prépare à la diplomatie ou, bien plus, qui lui est intrinsèquement liée. Elle intègre en outre le prélat ou le dignitaire dans des stratégies militaires ${ }^{60}$.

18 Un exemple significatif est celui de Durand de Tres Eminas, évêque de Marseille (1289-1312). Il provient lui aussi d'un milieu identique aux précédents, la familia de Bernard d'Anguissola ${ }^{61}$. Il reçoit d'importantes commissions de Nicolas IV et Boniface VIII ${ }^{62}$. Dès le retour de captivité de Charles II, Durand intègre l'entourage royal, sans doute comme conseiller, mais sans détenir de fonction administrative. Il veille sur les fondations royales de Notre-Dame de Nazareth d'Aix, devenue nécropole de Charles II en 1309, et du couvent de Saint-Maximin voué au culte dynastique de la Madeleine $^{63}$. Son action politique s'investit dans la fiscalité pontificale. Entre 1296 et 1304, il est collecteur de la décime alors levée pro negotio regni Sicilie. Il opère dans les provinces de Lyon, Vienne, Besançon, Tarentaise, Aix, Arles et Embrun, autrement dit le royaume d'Arles ${ }^{64}$. Tandis que Guillaume de Mandagout se voit simultanément confier celles de Lyon, Vienne, Tarentaise, Besançon et Embrun. Durand doit veiller à l'assignation des sommes destinées à l'armement de la flotte en $1296^{65}$. Sur ce plan, on peut lui rapprocher son collègue Geoffroi de Lincel, avocat et canoniste, prévôt d'Apt puis évêque de Gap, dont nous avons retracé ailleurs la carrière ${ }^{66}$.

19 La décime est une opération en soi diplomatique et militaire. Chez nos clercs, elle met en œuvre les compétences du négociateur, la délégation pontificale, le service de la monarchie. Elle contribue à la mobilisation du clergé, qu'il soit contribuable ou collecteur, dans la défense d'une monarchie vassale du pape. Elle concourt à intégrer l'épiscopat de Provence au sein d'une société politique, dont les premières assemblées à la fin du règne de Charles II manifestent les formes d'organisation primordiales ${ }^{67}$. 

cohésion. La formation intellectuelle y joue pour beaucoup, tout comme la reconnaissance de ses compétences par l'Église et l'État angevin. La collaboration avec l'une les met toujours en relation avec l'autre dans les terres angevines. Comme l'ont souligné les travaux sur les sacres royaux de 1266, 1289 et 130968, le modèle monarchique qui y est à l'œuvre ne saurait imposer à l'épiscopat de choisir entre le service du pape et celui du prince. Il s'inscrit pleinement dans le discours théocratique. Le cardinalat couronne la carrière de ceux qui vivent le plus longtemps et qui ont su constituer les réseaux de soutien les plus influents. Ces prélats savent mener à son terme les potentialités du sacerdoce, dont la fonction médiatrice et pacificatrice est mise au service de l'ordre politique. Elle s'exerce dans le domaine de la paix, par l'arbitrage, l'exécution de commissions pontificales, la négociation. Elle conduit le pasteur à occuper une place centrale dans la fiscalité pontificale, quand son affectation à la sauvegarde de la monarchie angevine ajoute encore à son rôle d'intermédiaire. En Provence, la médiation procède aussi d'une position géopolitique précise, cœur d'un dispositif entre capétiens, papauté, couronne d'Aragon, couronne de Sicile, qui préexiste à l'installation de la papauté en Avignon et qui a certes présidé au choix de ce lieu comme résidence pontificale.

\section{BIBLIOGRAPHIE}

\section{Sources manuscrites}

France, Digne-les-Bains, Arch. départementales des Alpes-de-Haute-Provence

$-1 G 25$

France, Marseille, Archives départementales des Bouches-du-Rhône

- 4G 1, no $12,21,22,65 ; 4 G 2, n^{\circ} 133,138 ; 4 G 3, n^{\circ} 283,291,304,317 ; 4 G 4, n^{\circ} 97 ; 4 G 5, n^{s} 160,161 ;$ $4 \mathrm{G} 6, \mathrm{n}^{\circ} 252 ; 4 \mathrm{G} 9, \mathrm{n}^{\circ} 223,224$;

$-5 \mathrm{G} 17, \mathrm{n}^{\circ} 114$

- B 1088;

Vatican, Archivio Apostolico Vaticano

- Instr. Misc. 359

Sources publiées

ALBANÈS, Joseph Hyacinthe - Histoire des évêques de Saint-Paul-Trois-Châteaux au quatorzième siècle. Corrections et documents. Montbéliard: Imprimerie de P. Hoffmann, 1885.

ALBANÈS, Joseph Hyacinthe - Gallia christiana novissima, Marseille. / d'après les documents authentiques recueillis dans les registres du Vatican et les archives locales par le chanoine J.-H. Albanès [...]; completée, annotée et publiée par le chanoine Ulysse Chevalier [...]. Marseille: P. Hoffmann (Montbéliard); impr. valentinoise (puis), 1899.

Medievalista, 28 | 2020 
ALBANÈS, Joseph Hyacinthe - Gallia christiana novissima, Arles / d'après les documents authentiques recueillis dans les registres du Vatican et les archives locales par le chanoine J.-H. Albanès [...]; completée, annotée et publiée par le chanoine Ulysse Chevalier [...]. Valence: P. Hoffmann (Montbéliard) / impr. valentinoise (puis), 1900.

ALBANÈS, Joseph Hyacinthe - Gallia christiana novissima, Avignon / par feu le chanoine J.-H. Albanès [...]; complétée, annotée et publiée par le chanoine Ulysse Chevalier [...]. Valence: impr. valentinoise, 1920.

BLANCARD, Louis - Documents inédits sur le commerce de Marseille au Moyen Âge. T. 2-4. Pièces commerciales diverses tirées des archives marseillaises du XIII ${ }^{e}$ siècle. Marseille: Typographie et Litographie Barlatier-Feissat Père et Fils, 1885.

BRESC, Henri (éd.) - La correspondance de Pierre Ameilh, archevêque de Naples puis d'Embrun (1363-1369). Paris: Éditions du Centre National de la Recherche Scientifique, 1972.

BUGHETTI, P. Benvenutus (éd.) - Processus canonizationis et legendœe variøe sancti Ludovici O. F. M. episcopi Tolosani, Analecta franciscana. T. 7. Quaracchi-Firenze: ex Typographia Collegii S. Bonaventurae, 1951.

CHEVALIER, Ulysse - Regeste dauphinois. Répertoire chronologique et analytique des documents imprimés et manuscrits relatifs à l'histoire du Dauphiné, des origines chrétiennes à l'an 1349. Valence: L'Imprimerie Valentinoise, 1913-1926.

DIGARD, Georges; FAUCON, Maurice; THOMAS, Antoine; FAWTIER, Robert (éd.) - Les registres de Boniface VIII : recueil des bulles de ce pape publiées ou analysées d'après les manuscrits originaux des Archives du Vatican. Paris: E. Thorin, 1907-1939, 4 vols. (BEFAR, $2^{\mathrm{e}}$ série, 4).

DOMÍNGUEZ SÁNCHEZ, Santiago (éd.) - Documentos de Bonifacio VIII (1294-1303) referentes a España. León: Universidad de León, 2006.

DOMÍNGUEZ SÁNCHEZ, Santiago(éd.) - Documentos de Nicolás IV (1288-1292) referentes a España. León: Universidad de León, 2009.

DOMÍNGUEZ SÁNCHEZ, Santiago (éd.) - Documentos de Martin IV (1281-1285) referentes a España. León: Universidad de León, 2010.

DOMÍNGUEZ SÁNCHEZ, Santiago (éd.) - Documentos de Clemente V (1305-1314) referentes a España. León: Universidad de León, 2014.

DOMÍNGUEZ SÁNCHEZ, Santiago (éd.) - Documentos de Honorio IV (1285-1287) referentes a España. León: Universidad de León, 2015.

FAILLON, M. - Monuments inédits sur l'apostolat de Sainte Marie Madeleine en Provence. T. 2. Paris: Jacques-Paul Migne, 1848.

FILANGIERI, Riccardo (éd.) - I registri della cancellaria angioina ricostruiti. Naples: Regia Cancellaria, 1950.

GRANDJEAN, Charles (éd) - Le registre de Benoît XI: recueil des bulles de ce pape publiées ou analysées d'après le manuscrit original des archives du Vatican. Paris: E. Thorin, 1905, 1 vol. (BEFAR, $2^{\mathrm{e}}$ série).

LANGLOIS, Ernest (éd.) - Les registres de Nicolas IV: recueil des bulles de ce pape publiées ou analysées d'aprés les manuscrits originaux des Archives du Vatican. Paris: E. Thorin, 1886-1892, 2 vols. (BEFAR, $2^{\mathrm{e}}$ série, 5).

MARTINI, A. (éd.) - ASV, Archivum Arcis, Arm. I-XVIII, 2177 et 2178. Cité du Vatican, 2008, dactylographié. 
PAPON, Jean-Pierre - Histoire générale de Provence. T. 3. Paris: L'Imprimerie de Ph. D. Pierres, 1784. PÉCOUT, Thierry (dir.); BERNARDI, Philippe; BONNAUD, Jean-Luc; CASSIOLI, Marco; MAILLOUX, Anne et al. (éd.) - L'enquête générale de Leopardo da Foligno dans le comté de Forcalquier (juin-septembre 1332). Paris: Comité des travaux historiques et scientifiques, 2017.

POMMEROL, Marie-Henriette Jullien de; MONFRIN, Jacques (éd.) - Bibliothèques ecclésiastiques au temps de la papauté d'Avignon. T. 2. Inventaires de prélats et de clercs français. Édition. Paris: CNRS éditions, 2001.

Regestum Clementis papae V [...]. nunc primum editi cura et studio monachrorum ordinis S. Benedicti [...]. Rome: ex Typogr. Vaticana, 1885-1892. 9 vols. et appendice.

RYMER, Thomas - Fœdera, conventiones, literce et cujuscumque generis acta publica, inter reges Anglice et alios. T. 1-2. Hagae comitis (apud Joannem Neauline), 1745.

SCARLATA, Marina (éd.) - Carte reali diplomatiche di Giacomo II d'Aragona (1291-1327) riguardanti l'Italia. Palermo: Società Siciliana per la Storia Patri, 1993.

SCHULTE, Johann Friedrich von - Die Geschichte der Quellen und Literatur des Canonischen Rechts von Papst Gregor IX. bis zum Concil von Trient. Stuttgart: Ferdinand Enke, 1877.

Études

ANDRETTA, Stefano; PEQUIGNOT, Stéphane; WAQUET, Jean-Claude (dir.) - De l'ambassadeur: les écrits relatifs à l'ambassadeur et à l'art de négocier du Moyen Âge au début du XIX ${ }^{e}$ siècle. Rome: École française de Rome, 2015.

BACHRACH, David S. - Religion and the Conduct of War, c. 300 - c. 1215. Woodbridge: Boydell Press, 2003.

BAGLIANI, Agostino Paravicini - I testamenti dei cardinali del duecento. Rome: Società alla Biblioteca Vallicelliana, 1980.

BAGLIANI, Agostino Paravicini - Boniface VIII. Un pape hérétique? Paris: Editions Payot \& Rivages, 2003.

BALBERGHE, Émile van - "Une copie exceptionnelle du Libellus de episcoporum electionibus de Guillaume de Mandagout”. in RAMAN, Anny; MANNING, Eugéne (éd.) - Miscellanea Martin Wittek. Album de codicologie et de paléographie offert à Martin Wittek. Louvain-Paris: Peters, 1993, pp.315-322. BEAUMONT, Jean-Pierre - Une famille de banquiers italiens, les Anguissola de Plaisance (première moitié du XIVe siècle). Chartres: École Nationale des Chartes, 1968. Thèse de Promotion.

BÉGOU-DAVIA, Michèle - “Guillaume de Mandagout”. in ARABEYRE, Patrick; HALPÉRIN, JeanLouis; KRYNEN, Jacques - Dictionnaire historique des juristes français, XII ${ }^{e}-X X^{e}$ siècle. Paris: Éditions PUF, 2007, p. 533.

BERTHE, Pierre-Marie - Les procureurs français à la cour pontificale d'Avignon, 1309-1376. Paris: Université Paris 4, 2014. Thèse de doctorat en Histoire médiévale.

BOESPFLUG, Thérese - La Curie au temps de Boniface VIII. Étude prosopographique. Rome: Istituto Storico Italiano per il Medioevo, 2005.

BONNOT-RAMBAUD, Isabelle (éd.) - Marseille et ses rois de Naples. La diagonale angevine (1265-1382). Marseille / Aix-en-Provence: Archives Municipales / Edisud, 1988.

BOYER, Jean-Paul - "Sacre et théocratie. Le cas des rois de Sicile Charles II (1289) et Robert (1309)”. Revue des Sciences philosophiques et théologiques 81/4 (1997), pp. 561-607. 
BOYER, Jean-Paul - "Le droit civil entre studium et cour de Naples". in BOYER, Jean-Paul; MAILLOUX, Anne; VERDON, Laure (dir.) - La justice temporelle dans les territoires angevins aux XIII et XIV siècles. Théories et pratiques (colloque d'Aix-en-Provence, 21-23 février 2002). Paris: École Française de Rome, 2005, pp. 47-82.

BRESC, Henri - "Les partis cardinalices et leurs ambitions dynastiques". in FAVIER, Jean; et al. (dir.) - Genèse et débuts du Grand Schisme d'Occident (actes du colloque international du CNRS, Avignon 25-28 septembre 1978). Paris: Éditions du Centre national de la recherche scientifique, 1980, pp. 45-57.

BRESC, Henri - "Marseille dans la guerre des Vêpres siciliennes". in BONNOT-RAMBAUD, Isabelle (éd.) - Marseille et ses rois de Naples. La diagonale angevine (1265-1382). Marseille / Aix-en-Provence: Archives Municipales / Edisud, 1988, pp. 43-49.

BRUNEL, Clovis - “Une table pascale de Guillaume de Mandagout”. Bibliothèque de l'École des chartes 84 (1923), pp. 161-165.

CADIER, Léon - Essai sur l'administration du royaume de Sicile sous Charles I ${ }^{\text {er }}$ et Charles II d'Anjou. Paris: E. Thorin, 1891.

CALENDINI, Paul - “Capre, Bx Rostang de”. in BAUDRILLART, Alfred ; VOGT, Albert ; ROUZIÈS, Urbain (dir.) - Dictionnaire d'histoire et de géographie ecclésiastiques. T. 11. Paris: Letouzey et Ané, 1949, col. 957-958.

CANTEAUT, Olivier; HÉLARY, Xavier; THÉRY, Julien (dir.) - 1314, une Europe en crise? La conjoncture politique européenne à la mort de Philippe le Bel (Université de Paris-Sorbonne, 2-4 octobre 2014), à paraître.

COLLARD, Franck ; COTTRET, Monique. (dir.) - Conciliation, réconciliation aux temps médiévaux et modernes [en ligne]. Nanterre: Presses universitaires de Paris Nanterre, 2012. [Consulté le 31 décembre 2015]. Accessible in https://doi.org/10.4000/books.pupo.2016.

COULET, Noël - "Un couvent royal: les Dominicaines de Notre-Dame-de-Nazareth d'Aix au XIII ${ }^{\mathrm{e}}$ s.”. Cahiers de Fanjeaux: Les Mendiants en Pays d'Oc au XIII siècle 8 (1973), pp. 233-262.

DÉLIVRÉ, Fabrice - “Le Libellus super electionibus de Guillaume de Mandagout (1286/1287). Histoire d'un succès dans l'Occident médiéval”. in GOERING, Joseph Ward; DUSIL, Stephan; THEIR, Andreas (dir.) - Proceedings of the Fourteenth International Congress of Medieval Canon Law, Toronto, 5-11 August 2012. Cité du Vatican: Biblioteca Apostolica Vaticana, 2016, pp. 233-242.

DÉLIVRÉ, Fabrice - “Mandagotus. Les avatars d'un traité juridique à succès". in BILOTTA, Maria Alesssandra (dir.) - Medieval Europe in Motion. Circulations juridiques et pratiques artistiques, intellectuelles et culturelles en Europe au Moyen Âge (XIII ${ }^{e}-X V^{e}$ siècle). Lisbonne, 25-25 février 2016, à paraître.

DOMÍNGUEZ SÁNCHEZ, Santiago - Los procuradores de los reinos hispánicos ante la curia romana en el siglo XIII. León: Universidad de León, 2007.

DOSSAT, Yves - “L'Université de Toulouse, Raymond VII, les Capitouls et le roi". Cahiers de Fanjeaux: Les Universités du Languedoc au XIII e siècle 5 (1970), pp. 58-91.

DROCOURT, Nicolas - "Ambassadeurs étrangers à Constantinople: moyens de contacts, d'échanges et de connaissances partielles du monde byzantin (VIII ${ }^{\mathrm{e}}$-XII ${ }^{\mathrm{e}}$ siècles)". in CLÉMENT, François; TOLAN, John; WILGAUX, Jérôme (dir.) - Espaces d'échanges en Méditerranée. Antiquité et Moyen Âge [en ligne]. Rennes: Presses universitaires de Rennes, 2006, pp. 107-134. [Consulté le 31 décembre 2015]. Accessible in https://doi.org/10.4000/books.pur.7841. 
FERRER MALLOL, María Teresa; MOEGLIN, Jean-Marie; PÉQUIGNOT, Stéphane; SÁNCHEZ MARTÍNEZ, Manuel (eds.) - Negociar en la Edad Media - Négocier au Moyen Âge. Barcelone: CSIC, 2005.

GALLAND, Bruno - "Les hommes de culture dans la diplomatie pontificale au XIII ${ }^{\mathrm{e}}$ siècle". Mélanges de l'École française de Rome. Moyen Âge, Temps modernes 108/2 (1996), pp. 615-643.

GALLO, Alexandra - "Le développement d'un réseau diplomatique par le conseil de ville de Sisteron au XIV ${ }^{\mathrm{e}}$ siècle". in Les relations diplomatiques au Moyen Âge. Formes et enjeux [en ligne]. Paris: Éditions de la Sobornne, 2011, pp. 219-225 [Consulté le 31 décembre 2015]. Accessible in https://doi.org/10.4000/books.psorbonne.16469.

GILLES, Henri - "Le traité de la coutume de Guillaume de Ferrières". in GILLES, Henri - Université de Toulouse et enseignement du droit, XIII ${ }^{e}-X I^{e}$ siècles. Toulouse: Presses de l'Université Toulouse I Capitole, 1992, pp. 127-138.

GIORDANENGO, Gérard - Lexikon des Mittelalters. T. 4. Erzkanzler bis Hiddensee. München. Zürich: Artemis, 1989.

GIORDANENGO, Gérard - "Ferrières, Pierre de". in ARABEYRE, Patrick; HALPÉRIN, Jean-Louis; KRYNEN, Jacques - Dictionnaire historique des juristes français, XII ${ }^{e} X^{e}$ siècle. Paris: Éditions PUF, 2007, pp. 327-328.

GIUNTA, Francesco; CORRAO, Pietro (dir.) - La società mediterranea all'epoca del Vespro, $11^{\circ}$ Congresso di Storia della Corona d'Aragona, Palermo, Trapani, Erice, 23-30 aprile 1982. Palerme: Accademia di scienze lettere e arti, 1983-1984.

GRAVA, Yves - "Les ambassades provençales au XIV e siècle et les enjeux de la communication”. in Actes des congrès de la Société des historiens médiévistes de l'enseignement supérieur public, $24^{\mathrm{e}}$ congrès, Avignon. La circulation des nouvelles au Moyen Âge. Paris-Rome: Éditions de la Sorbonne, École française de Rome, 1994, pp. 25-36.

GUENÉE, Benard - Entre l'Église et l'État. Quatre vies de prélats français à la fin du Moyen Âge (XIII XVe siècles). Paris: Gallimard, 1987.

GUILLEMAIN, Bernard - "Le milieu épiscopal et cardinalice de Bernard Gui". Cahiers de Fanjeaux: Bernard Gui et son monde 16 (1981), pp. 317-332.

HÉBERT, Michel - "Une identité mise en scène: les premières assemblées représentatives dans les comtés de Provence et de Forcalquier (XIII ${ }^{\mathrm{e}}$-début XIV ${ }^{\mathrm{e}}$ siècles)". in BOYER, Jean-Paul; MAILLOUX, Anne; VERDON, Laure (dir.) - Identités angevines. Entre Provence et Naples (Aix-en-Provence, 20-22 octobre 2011). Aix-en-Provence: Presses universitaires de Provence, 2016, pp. 183-194.

HOUSLEY, Norman - The Italian Crusades. The Papal-Angevin Alliance and the Crusades against Christian Lay Powers, 1254-1343. Oxford: Claredon Press, 1982.

JUGIE, Pierre - "L'activité diplomatique du cardinal Gui de Boulogne en France au milieu du XIVe siècle”. Bibliothèque de l'École des chartes 145/1 (1987), pp. 99-127.

KIESEWETTER, Andreas - "Karl II. von Anjou, Marseille und Neapel”. in BONNOT-RAMBAUD, Isabelle (dir.) - Marseille et ses rois de Naples. La diagonale angevine (1265-1382). Marseille / Aix-enProvence: Archives Municipales / Edisud, 1988, pp. 61-75.

KIESEWETTER, Andreas - "La cancelleria angioina”. in L'État angevin. Pouvoir, culture et société entre XIII et XIV siècle. Actes du colloque international, Rome-Naples, novembre 1995. Paris-Rome: École Française de Rome, 1998, pp. 361-415. 
LAZZARINI, Isabella - “À propos de diplomatie médiévale: pratiques, modèles et langages de la négociation en Italie (XIV -XV siècles)”. Médiévales [en ligne] 74 (2018), pp. 133-154 [Consulté le 31 décembre 2019]. Accessible in https://doi.org/10.4000/medievales.8653.

LE ROUX, Amandine - "Mise en place des collecteurs et des collectories dans le royaume de France et en Provence (1316-1378)". Lusitania sacra. O papado de Avinhão nos reinos do Ocidente 22 (2010), pp. 45-62.

LE ROUX, Amandine - "Des collecteurs spécialisés aux collecteurs généralistes, l'établissement du système collectoral en Provence (1249-1514)". in MORELLO BAGET, Jordi (dir.) - Financiar el reino terrenal. La contribución de la Iglesia a finales de la Edad Media (s. XIII-XVI). Barcelone: CSIC, 2013, pp. 107-129.

LE ROUX, Amandine - "L'écrit, un outil de gouvernement financier de la papauté. L'exemple de la correspondance entre la papauté et ses collecteurs". in BÉRENGER, Agnès; DARD, Olivier (éd.) Gouverner par les lettres, de l'Antiquité à l'époque contemporaine. Actes du colloque de Metz, 10-12 octobre 2013. Metz: Centre de Recherche Universitaire Lorrain d'Histoire, 2015, pp. 287-311.

LE ROUX, Amandine - "Le recouvrement de la décime par les collecteurs pontificaux de 1316 à 1503 (royaume de France et Provence)”. in BALOUP, Daniel; SÁNCHEZ MARTÍNEZ, Manuel (dir.) Partir en croisade à la fin du Moyen Âge. Financement et logistique. Toulouse: Presses Universitaires du Midi, 2015, pp. 55-80.

LE ROUX, Amandine - "Pratiques comptables du gouvernement pontifical. L'histoire scripturale des comptes des collecteurs pontificaux provençaux (1274-1406)". Comptabilités [en ligne] 10 (2019) [Consulté le 31 décembre 2019]. Accessible in https://journals.openedition.org/ comptabilites/2545.

MALECZEK, Werner - “Die Urkunden des päpstlichen Legaten Johannes Boccamazza, Kardinalbischofs von Tusculum, aus den Jahren 1286 und 1287 (Legation ins Reich in der Spätzeit König Rudolfs von Habsburg)". Archiv für Diplomatik. Schriftgeschichte, Siegel- und Wappenkunde 59 (2013), pp. 35-132.

MEIJERS, Eduard Maurits - "La première époque d'épanouissement de l'enseignement du droit à l'université de Toulouse (1280-1330)". in MEIJERS, Eduard Maurits; FEENSTRA, Robert; FISCHER, Herman Frederik Willem Daniel (éd.) - Études d'histoire du droit. T. 3. Le droit romain au Moyen Âge. Leyde: Universitaire Pers Leiden, 1959, pp. 167-208.

MINIERI-RICCIO, Camillo - Genealogia di Carlo I d'Angiò, prima generazione. Naples: Stabilimento Tipografico di Vincenzo Priggioba, 1857.

MISCHLEWSKI, Adalbert - Un ordre hospitalier au Moyen Âge. Les chanoines réguliers de Saint-Antoineen-Viennois. Grenoble: Presses Universitaires de Grenoble, 1995.

MOLLAT, Guillaume - “Guillaume de Mandagout”. in NAZ, Raoul (dir.) - Dictionnaire de droit canonique. T. 5. Paris: Letouzey ey Ané, 1953, col. 1077-1078.

MONTI, Gennaro Maria - "Carlo II e i debiti angioino verso la Santa Sede”. in MONTI, Gennaro Maria - Da Carlo I a Roberto d'Angiò. Ricerche e documenti. Trani: Vecchi, 1936, pp. 117-132.

MOREMBERT, Henri Tribout de - “Capre, Rostang de”. in PRÉVOST, Michel; D’AMAT, Jean-Charles Roman - Dictionnaire de biographie française. T. 7. Paris: Letouzey et Ané, 1956, col. 1081.

MOREMBERT, Henri Tribout de - "Ferrières, Pierre de". in BAUDRILLART, Alfred ; VOGT, Albert ; ROUZIÈS, Urbain (dir.) - Dictionnaire d'histoire et de géographie ecclésiastique. T. 16. Paris: Letouzey et Ané, 1967, col. 1287-1288. 
OTCHAKOVSKY-LAURENS, François - "Le statut des ambassadeurs marseillais au regard des pratiques diplomatiques au XIV ${ }^{\mathrm{e}}$ siècle". in La diplomatie des villes au Moyen Âge et au premier âge moderne, XII ${ }^{e}-\mathrm{XVI}^{e}$ siècle (actes du colloque international, Montpellier, 17-18 novembre 2017), à paraître.

PALMIERI, Stefano - La cancelleria del regno di Sicilia in età angioina. Naples: M. D'Auria Editore, 2006.

PÉCOUT, Thierry - "Une technocratie au service d'une théocratie. Culture et formation intellectuelle des évêques de Provence (milieu du XIII ${ }^{\mathrm{e}}$ siècle - milieu du XIV ${ }^{\mathrm{e}}$ siècle)". in CÉVINS, Marie-Madeleine; MATZ, Jean-Michel (dir.) - Formation intellectuelle et culture du clergé dans les territoires angevins (milieu du XIII ${ }^{e}$-fin du XV siècle), colloque d'Angers, 15-16 novembre 2002. Paris: École Française de Rome, 2005, pp. 95-116.

PÉCOUT, Thierry - "Diplômes, diplomates et diplomatie: le registre du maître rational Jean de Revest, procureur de la reine Jeanne in Romana Curia, 1343-1347". in KORDÉ, Zoltán; PETROVICS, István (dir.) - La diplomatie des États angevins aux XIII ${ }^{e}$ et XIV ${ }^{e}$ siècles, Actes du colloque international de Szeged, Visegràd et Budapest, 13-16 septembre 2007. Rome-Szeged: Accademia d'Ungheria in Roma, 2010, pp. 251-287.

PÉCOUT, Thierry - Ultima ratio. Vers un État de raison. L'épiscopat, les chanoines et le pouvoir des années 1230 au début du XIV ${ }^{e}$ siècle (provinces ecclésiastiques d'Arles, Aix et Embrun). Paris: Université de Paris I, 2011. Mémoire d'Habilitation à diriger les recherches.

PÉCOUT, Thierry - “Jacques Duèze, évêque de Fréjus (1300-1310)”. Cahiers de Fanjeaux: Jacques Duèze-Jean XXII et le Midi 45 (2013), pp. 41-68.

PÉCOUT, Thierry - “Épiscopat et papauté en Provence: une refondation”. Cahiers de Fanjeaux: Innocent III et le Midi 50 (2015), pp. 419-452.

PÉCOUT, Thierry - "Les évêques de Gap, XII ${ }^{\mathrm{e}}-\mathrm{XIV}{ }^{\mathrm{e}}$ siècle: les enjeux d'un contrôle politique entre Provence et Dauphiné”. in PLAYOUST, Pierre-Yves (dir.) - Gap et les territoires gapençais de la préhistoire à nos jours. Grenoble: Presses universitaires de Grenoble, 2016, pp. 15-53.

PÉCOUT, Thierry - “Aux origines d'une culture administrative: le clergé des cathédrales et la genèse d'une comptabilité princière en Provence à la fin du XIII ${ }^{\mathrm{e}}$ siècle”. in PÉCOUT, Thierry (dir.) - De l'autel à l'écritoire. Aux origines des comptabilités princières en Occident (XII ${ }^{e}$-XIVe siècle), Actes du colloque international d'Aix-en-Provence, 13-14 juin 2013. Paris: De Boccard, 2017, pp. 49-67.

PÉCOUT, Thierry - "Docteurs et professeurs de droit civil parmi les grands officiers de Provence angevine, 1246-1343". in MATHIEU, Isabelle; MATZ, Jean-Michel (dir.) - Formations et cultures des officiers et de l'entourage des princes dans les territoires angevins (milieu XIII ${ }^{e}$-fin XV $\mathrm{XV}^{e}$ siècle) - Percorsi di formazione e culture degli ufficiali e dell'entourage dei principi nei territori angioini (metà XIII-fine XV secolo) [en ligne]. Rome: Publications de l'École Française de Rome, 2019, pp. 119-142 [Consulté le 31 décembre 2019]. Accessible in https://doi.org/10.4000/books.efr.4047.

PÉQUIGNOT, Stéphane - Au nom du roi. Pratique diplomatique et pouvoir durant le règne de Jacques II d'Aragon (1291-1327). Madrid: Casa de Velázquez, 2009.

REYNAUD, Marcelle-Renée - "La politique de la Maison d'Anjou et la soustraction d'obédience en Provence (1398-1402)". Cahiers d'histoire 24 (1979), pp. 45-57.

RUSSEL, Frederick H. - The Just War in the Middle Ages. Cambridge: Cambridge University Press, 1975.

SALAVERT Y ROCA, Vicente - Cerdeña y la expansiòn mediterrànea de la corona de Aragòn (1297-1314). Madrid: CSIC, 1956. 
SAXER, Victor - "Les ossements dits de sainte Marie-Madeleine conservés à Saint-Maximin-laSainte-Baume”. Provence historique 27 (1977), pp. 257-302.

SOCIÉTÉ DES HISTORIENS MÉDIÉVISTES DE L'ENSEIGNEMENT SUPÉRIEUR PUBLIC (FRANCE),</

\section{NOTES}

1. PÉCOUT, Thierry - "Diplômes, diplomates et diplomatie: le registre du maître rational Jean de Revest, procureur de la reine Jeanne in Romana Curia, 1343-1347". in Kordé, Zoltán; PETROVICS, István (dir.) - La diplomatie des États angevins aux XIIIe et XIVe siècles, Actes du colloque international de Szeged, Visegràd et Budapest, 13-16 septembre 2007. Rome-Szeged: Accademia d'Ungheria in Roma, 2010, pp. 251-287.

2. La négociation à la Curie et les réseaux cardinalices qui la sous-tendent ont fait l'objet de travaux précurseurs, à la suite des ouvrages classiques sur la légation: BRESC, Henri (éd.) - La correspondance de Pierre Ameilh, archevêque de Naples puis d'Embrun (1363-1369). Paris: Éditions du Centre national de la recherche scientifique, 1972; BRESC, Henri - "Les partis cardinalices et leurs ambitions dynastiques". in FAVIER, Jean; et al. (dir.) - Genèse et débuts du Grand Schisme d'Occident (actes $d u$ colloque international du CNRS, Avignon 25-28 septembre 1978). Paris: Éditions du Centre national de la recherche scientifique, 1980, pp. 45-57; GALLAND, Bruno - "Les hommes de culture dans la diplomatie pontificale au XIII ${ }^{\mathrm{e}}$ siècle". Mélanges de l'École française de Rome. Moyen Âge, Temps modernes 108/2 (1996), pp. 615-643; JUGIE, Pierre - "L'activité diplomatique du cardinal Gui de Boulogne en France au milieu du XIV siècle". Bibliothèque de l'École des chartes 145/1 (1987), pp. 99-127. Tout particulièrement, la dernière génération de recherches insiste sur les méthodes et le personnel de la négociation. FERRER MALLOL, María Teresa; MOEGLIN, Jean-Marie; PÉQUIGNOT, Stéphane; SÁNCHEZ MARTÍNEZ, Manuel (eds.) - Negociar en la Edad Media - Négocier au Moyen Âge. Barcelone: CSIC, 2005; DROCOURT, Nicolas - "Ambassadeurs étrangers à Constantinople: moyens de contacts, d'échanges et de connaissances partielles du monde byzantin (VIII ${ }^{\mathrm{e}}$-XII ${ }^{\mathrm{e}}$ siècles)". in CLÉMENT, François; TOLAN, John; WILGAUX, Jérôme (dir.) Espaces d'échanges en Méditerranée. Antiquité et Moyen Âge [en ligne]. Rennes: Presses universitaires de Rennes, 2006, pp.107-134. [Consulté le 31 décembre 2015]. Accessible in https://doi.org/ 10.4000/books.pur.7841; DOMÍNGUEZ SÁNCHEZ, Santiago - Los procuradores de los reinos hispánicos ante la curia romana en el siglo XIII. León: Universidad de León, 2007; VALÉRIAN, Dominique - "Les agents de la diplomatie des souverains maghrébins avec le monde chrétien (XII ${ }^{\mathrm{e}}-\mathrm{XV}^{\mathrm{e}}$ siècle)". Anuario de Estudios Medievales 38/2 (2008), pp. 985-900; PÉQUIGNOT, Stéphane - Au nom du roi. Pratique diplomatique et pouvoir durant le règne de Jacques II d'Aragon (1291-1327). Madrid: Madrid: Casa de Velázquez, 2009; SOCIÉTÉ DES HISTORIENS MÉDIÉVISTES DE L'ENSEIGNEMENT SUPÉRIEUR PUBLIC (FRANCE), Congrès - Les relations diplomatiques au Moyen Âge. Formes et enjeux. Paris: Publications de la Sorbonne, 2011; COLLARD, Franck; COTTRET, Monique (dir.) Conciliation, réconciliation aux temps médiévaux et modernes [en ligne]. Nanterre: Presses universitaires de Paris Nanterre, 2012. [Consulté le 31 décembre 2015]. Accessible in https:// doi.org/10.4000/books.pupo.2016; BERTHE, Pierre-Marie - Les procureurs français à la cour pontificale d'Avignon, 1309-1376. Paris: Université Paris 4, 2014. Thèse de doctorat en Histoire médiévale; VALLERY-RADOT, Sophie - "La diplomatie menée par l'ambassade du roi de France au Concile de Constance". in SIGNORI, Gabriela; STUDT, Birgit (dir.) - Das Konstanzer Konzil als europäisches Ereignis: Begegnungen, Medien und Rituale [en ligne] 79 (2014), pp. 89-106 [Consulté le 31 décembre 2015]. Accessible in https://doi.org/10.11588/vuf.2014.0.41846; ANDRETTA, Stefano; PEQUIGNOT, Stéphane; WAQUET, Jean-Claude (dir.) - De l'ambassadeur: les écrits relatifs à l'ambassadeur et à l'art de négocier du Moyen Âge au début du XIXe siècle. Rome: École française de Rome, 2015; LAZZARINI, Isabella - “À propos de diplomatie médiévale: pratiques, modèles et 
langages de la négociation en Italie (XIV ${ }^{\mathrm{e}}-\mathrm{XV}^{\mathrm{e}}$ siècles)". Médiévales [en ligne] 74 (2018), pp. 133-154 [Consulté le 31 décembre 2019]. Accessible in https://doi.org/10.4000/medievales.8653. L'édition scientifique s'est également orientée vers une diplomatique de la diplomatie: SCARLATA, Marina (éd.) - Carte reali diplomatiche di Giacomo II d'Aragona (1291-1327) riguardanti l'Italia. Palermo: Società Siciliana per la Storia Patri, 1993. Ces processus s'insèrent dans une lente construction des conditions juridiques de l'état de belligérance, que l'on doit aux canonistes, ainsi que de ses rites: RUSSEL, Frederick H. - The Just War in the Middle Ages. Cambridge: Cambridge University Press, 1975; BACHRACH, David S. - Religion and the Conduct of War, c. 300 - c. 1215. Woodbridge: Boydell Press, 2003.

3. Sur ces enjeux européens, on pourra se rapporter à deux actes de colloques: GIUNTA, Francesco; CORRAO, Pietro (dir.) - La società mediterranea all'epoca del Vespro, $11^{\circ}$ Congresso di Storia della Corona d'Aragona, Palermo, Trapani, Erice, 23-30 aprile 1982. Palerme: Accademia di Scienze Lettere e Arti, 1983-1984; CANTEAUT, Olivier, HÉLARY, Xavier, THÉRY, Julien (dir.) - 1314, une Europe en crise? La conjoncture politique européenne à la mort de Philippe le Bel (Université de ParisSorbonne, 2-4 octobre 2014), à paraître. Sur les négociations propres à l'empire : MALECZEK, Werner - "Die Urkunden des päpstlichen Legaten Johannes Boccamazza, Kardinalbischofs von Tusculum, aus den Jahren 1286 und 1287 (Legation ins Reich in der Spätzeit König Rudolfs von Habsburg)”. Archiv für Diplomatik. Schriftgeschichte, Siegel- und Wappenkunde 59 (2013), pp. 35-132.

4. PÉCOUT, Thierry - "Épiscopat et papauté en Provence: une refondation". Cahiers de Fanjeaux: Innocent III et le Midi 50 (2015), pp. 419-452.

5. PÉCOUT, Thierry - Ultima ratio. Vers un État de raison. L'épiscopat, les chanoines et le pouvoir des années 1230 au début du XIVe siècle (provinces ecclésiastiques d'Arles, Aix et Embrun). Paris: Université de Paris I, 2011. Mémoire d'Habilitation à diriger les recherches. PÉCOUT, Thierry - "Une technocratie au service d'une théocratie. Culture et formation intellectuelle des évêques de Provence (milieu du XIII ${ }^{\mathrm{e}}$ siècle - milieu du XIV siècle)". in CÉVINS, Marie-Madeleine; MATZ, Jean-Michel (dir.) - Formation intellectuelle et culture du clergé dans les territoires angevins (milieu du $X I I I^{e}-$ fin du XV siècle), colloque d'Angers, 15-16 novembre 2002. Rome: École française de Rome, 2005, pp. 95-116. Les travaux sur le rôle des "hommes de culture" dans les activités diplomatiques ont tendance à privilégier le milieu cardinalice plutôt que l'épiscopat: GALLAND, Bruno - "Les hommes de culture dans la diplomatie pontificale au XIII siècle" ..., pp. 615-643. Au sujet de la Provence angevine, les recherches concernant les échanges diplomatiques ont fait la part belle aux villes, mais le clergé séculier a peu attiré l'attention: GRAVA, Yves - "Les ambassades provençales au XIV ${ }^{\mathrm{e}}$ siècle et les enjeux de la communication". in Actes des congrès de la Société des historiens médiévistes de l'enseignement supérieur public, 24e congrès, Avignon. La circulation des nouvelles au Moyen Âge. Paris-Rome: Éditions de la Sorbonne, École française de Rome, 1994, pp. 25-36; REYNAUD, Marcelle-Renée - "La politique de la Maison d'Anjou et la soustraction d'obédience en Provence (1398-1402)". Cahiers d'histoire 24 (1979), pp. 45-57; GALLO, Alexandra - "Le développement d'un réseau diplomatique par le conseil de ville de Sisteron au XIV ${ }^{\mathrm{e}}$ siècle". in Les relations diplomatiques au Moyen Âge. Formes et enjeux [en ligne]. Paris: Éditions de la Sobornne, 2011, pp. 219-225 [Consulté le 31 décembre 2015]. Accessible in https://doi.org/ 10.4000/books.psorbonne.16469; OTCHAKOVSKY-LAURENS, François - "Le statut des ambassadeurs marseillais au regard des pratiques diplomatiques au XIV ${ }^{\mathrm{e}}$ siècle". in La diplomatie des villes au Moyen Âge et au premier âge moderne, XII ${ }^{e}-X V I^{e}$ siècle (actes du colloque international, Montpellier, 17-18 novembre 2017), à paraître.

6. Ou plutôt Andrea d'Anguissola. Anguissola, d'une famille d'origine placentine, plutôt que Languissel, patronyme francisé habituellement utilisé par l'historiographie. BEAUMONT, JeanPierre - Une famille de banquiers italiens, les Anguissola de Plaisance (première moitié du XIVe siècle). Chartres: École Nationale des Chartes, 1968. Thèse de la Promotion.

7. PÉCOUT, Thierry - "Diplômes, diplomates et diplomatie: le registre du maître rational Jean de Revest, procureur de la reine Jeanne in Romana Curia, 1343-1347” ..., pp. 251-287. 
8. MEIJERS, Eduard Maurits - "La première époque d'épanouissement de l'enseignement du droit à l'université de Toulouse (1280-1330)". in MEIJERS, Eduard Maurits; FEENSTRA, Robert; FISCHER, Herman Frederik Willem Daniel (éd.) - Études d'histoire du droit. T. 3. Le droit romain au Moyen Âge. Leyde: Universitaire Pers Leiden, 1959, pp. 167-208; GILLES, Henri - "Le traité de la coutume de Guillaume de Ferrières". in GILLES, Henri - Université de Toulouse et enseignement du droit, XIII ${ }^{e}$ XVI siècles. Toulouse; Presses de l'Université Toulouse I Capitole, 1992, pp. 127-138, qui place ses études à Bologne; DOSSAT, Yves - "L'Université de Toulouse, Raymond VII, les Capitouls et le roi”. Cahiers de Fanjeaux: Les Universités du Languedoc au XIII ${ }^{e}$ siècle 5 (1970), pp. 75-76; CADIER, Léon - Essai sur l'administration du royaume de Sicile sous Charles Ir et Charles II d'Anjou. Paris: E. Thorin, 1891, p. 240; GIORDANENGO, Gérard - "Ferrières, Guillaume de". in ARABEYRE, Patrick; HALPÉRIN, Jean-Louis; KRYNEN, Jacques - Dictionnaire historique des juristes français, $\mathrm{XII}^{e}$-XXe ${ }^{e}$ siècle. Paris: Éditions PUF, 2007, p. 327.

9. ALBANÈS, Joseph Hyacinthe - Gallia christiana novissima, Marseille. / d'après les documents authentiques recueillis dans les registres du Vatican et les archives locales par le chanoine J.-H. Albanès [...]; completée, annotée et publiée par le chanoine Ulysse Chevalier [...]. Marseille: P. Hoffmann (Montbéliard); impr. valentinoise (puis), 1899 [désormais GCNN, Marseille], col. 771-777; le 11 août 1291, la provision pontificale ajoute à sa prévôté la perception des revenus des églises de SaintAnatole et Causideriis au diocèse de Toulon, attachées à sa prébende, ce qui nécessite l'ordination de Guillaume par l'évêque de Marseille, à laquelle il s'engage pour l'année suivant sa provision, ce qui lui permet d'obtenir dispense pour en percevoir les fruits (L Nicolas IV, $\mathrm{n}^{\circ} 5823$; GCNN, Marseille, $\mathrm{n}^{\circ}$ 1272).

10. KIESEWETTER, Andreas - "La cancelleria angioina". in L'État angevin. Pouvoir, culture et société entre XIII ${ }^{e}$ et XIV ${ }^{e}$ siècle. Actes du colloque international, Rome-Naples, novembre 1995. Paris-Rome: École Française de Rome, 1998, p. 383.

11. GCNN, Marseille, $\mathrm{n}^{\circ} 1276$. FILANGIERI, Riccardo (éd.) - I registri della cancellaria angioina ricostruiti. Naples: Regia Cancellaria, 1950 sqq. [désormais RCA], t. 39, n 10-12, pp. 12-18, 23-27 avril 1291; RCA, t. 49, no 99, pp. 59; RCA, t. 49, nº 181, pp. 101; RCA, t. 47, n 580, pp. 207; RCA, t. $47, \mathrm{n}^{\circ} 722$, pp. 248. RCA, t. 48, n $\mathrm{n}^{\circ} 49, \mathrm{pp} .34,4$ février 1294 , concession à Philippe de la principauté de Tarente; RCA, t. 48, $\mathrm{n}^{\circ}$ 45, pp. 29, 5 février 1294, en faveur de Tommaso Scillato de Salerno; RCA, t. 48, $\mathrm{n}^{\circ} 13$, pp. 11, 5 janvier 1294, en faveur d'Othon de Tucziaco.

12. Le 8 mars il est encore à Arles (GCNN, Marseille, $\left.n^{\circ} 1276\right)$ et le 27 juillet il est de retour à Aix.

13. Parmi les nominations du 18 septembre 1294, la quasi-totalité des promus est proche du roi angevin. Le cardinal Latino Malabranca (OP), neveu de Nicolas III, doyen du Sacré Collège est rallié par Charles II, mais il décède dès août 1294. Guglielmo Longhi († 1319), créé cardinal diacre par Célestin V le 18 septembre à l'instigation de Charles II, devient quant à lui son chancelier vers 1294-1295. Voir aussi le Sacré Collège lors du conclave de juillet 1294: ASV, Archivum Arcis, Arm. IXVIII, 2177 et 2178 (édités par A. Martini, Cité du Vatican, 2008, dactylographié); T RINCI, Annalaura - "Il collegio cardinalizio di Celestino V". in CAPEZZALI, Walter (dir.) - Celestino V e i suoi tempi: realtà spirituale et realtà politica. L'Aquila: Centro Celestiniano/Sezione storica, 1990, pp. 19-34; PALMIERI, Stefano - La cancelleria del regno di Sicilia in età angioina. Naples: M. D'Auria Editore, 2006, p. 168.

14. L Boniface VIII, $\mathrm{n}^{\circ}$ 227. L Boniface VIII, $\mathrm{n}^{\circ}$ 797: prévôté, canonicat et prébende de Marseille, revenus des églises de Saint-Anatole et de Causideriis.

15. Allusion à son incapacité pour maladie, dans une lettre du 19 septembre 1295: LCu Boniface VIII, $\mathrm{n}^{\circ}$ 827. Guillaume avait reçu licence de tester le 11 juillet 1295 , mais son testament ne nous est pas parvenu: BAGLIANI, Agostino Paravicini - I testamenti dei cardinali del duecento. Rome: Società alla Biblioteca Vallicelliana, 1980, p. 59.

16. SALAVERT Y ROCA, Vicente - Cerdeña y la expansiòn mediterrànea de la corona de Aragòn (1297-1314). Madrid: CSIC, 1956, pp. 47-117, jusqu'au traité d'Agnani. 
17. MOREMBERT, Henri Tribout de - “Capre, Rostang de”. in PRÉVOST, Michel; D’AMAT, JeanCharles Roman - Dictionnaire de biographie française. T. 7. Paris: Letouzey et Ané, 1956, col. 1081; CALENDINI, Paul - "Capre, Bx Rostang de". in BAUDRILLART, Alfred ; VOGT, Albert ; ROUZIÈS, Urbain (dir.) - Dictionnaire d'histoire et de géographie ecclésiastiques. T. 11. Paris: Letouzey et Ané, 1949, col.957-958; ALBANÈS, Joseph Hyacinthe - Gallia christiana novissima, Arles / d'après les documents authentiques recueillis dans les registres $d u$ Vatican et les archives locales par le chanoine J.-H. Albanès [...]; completée, annotée et publiée par le chanoine Ulysse Chevalier [...]. Valence: P. Hoffmann (Montbéliard) / impr. valentinoise (puis), 1900 [désormais GCNN, Arles], nº 1301, 8 avril 1278. Marseille, Archives départementales des Bouches-du-Rhône [désormais AD13], 4G 6, $\mathrm{n}^{\circ} 252$ et GCNN, Arles, $\mathrm{n}^{\circ} 1302,4$ juin 1278. GCNN, Arles, $\mathrm{n}^{\circ} 1280,29$ janvier 1285. Avant son épiscopat, Rostaing apparaît comme chanoine (AD13, 4G $1, \mathrm{n}^{\circ} 12,12$ novembre 1284 , et $4 \mathrm{G} 9, \mathrm{n}^{\circ} 224$, 13 octobre 1285), mais beaucoup plus fréquemment avec son titre d'ouvrier: AD13, 4G 3, $\mathrm{n}^{\circ} 283$, 8 avril 1276; 4G 3, n ${ }^{\circ} 317,9$ février 1284; 4G 2, nos 133 et 138, 29 avril 1284; 4G 3, n 291,29 avril 1284; $4 \mathrm{G} 5, \mathrm{n}^{\text {os }} 160-161,29$ avril 1284 et 16 novembre $1284 ; 4 \mathrm{G} 1, \mathrm{n}^{\text {os }} 65,21$ et 22 , les 2,12 et 16 novembre 1284; $4 \mathrm{G} 3, \mathrm{n}^{\circ} 304$ et $4 \mathrm{G} 9, \mathrm{n}^{\circ} 223,12$ novembre $1284 ; 4 \mathrm{G} 4, \mathrm{n}^{\circ} 97,20$ juillet 1286.

18. GCNN, Arles, $\mathrm{n}^{\circ} 1332$ et L Nicolas IV, $\mathrm{n}^{\circ}$ 587. Le chapitre, après la mort de Bertrand Amalrici, a désigné trois compromissaires qui se sont accordés sur son nom, le précenteur Raymond Saboni, le prieur claustral Raymond de Coirano et Raymond de Auraga, prieur de Châteauneuf, ce dernier l'emportant en cas de désaccord des trois. Rostaing est pourvu avec le conseil des cardinaux Girolamo Masci OFM, évêque de Palestrina, Gervais Jeancolet de Clinchamp, du titre de San Martino in Montibus, et Benedetto Caetani, diacre de San Niccolò in Carcere Tulliano.

19. GCNN, Arles, $\mathrm{n}^{\circ} 1333-1334,19-20$ septembre 1286. GCNN, Arles, $\mathrm{n}^{\circ} 1340$ LCu Nicolas IV, $\mathrm{n}^{\circ} 583$, 3 mai 1288; $\mathrm{n}^{\circ} 1342$ et L Nicolas IV, $\mathrm{n}^{\circ}$ 601-603, 12 août 1288. L Nicolas IV, $\mathrm{n}^{\circ} 1095$ et GCNN, Arles, $\mathrm{n}^{\circ} 1345$ et $L$ Nicolas IV, $\mathrm{n}^{\circ} 1095$ et 1136, 23 juillet 1289, sur les versements au sénéchal de Provence. 20. $R C A$, t. $49, \mathrm{n}^{\circ} 167,22$ février 1294 , si l'on en croit la titulature du prévôt de Grasse $\left(\mathrm{n}^{\circ} 166\right)$ bénéficiant de la même protection et sur la lettre duquel celle-ci est copiée.

21. GCNN, Arles, nos 1347-1348. MINIERI-RICCIO, Camillo - Genealogia di Carlo I d'Angiò, prima generazione. Naples: Stabilimento Tipografico di Vincenzo Priggioba, 1857, n⿳0 38, pp. 176-178: Charles II se rend au col de Panissars, mais le roi d'Aragon ne s'y trouve pas; un procès-verbal est alors dressé, auxquels souscrivent notamment Albert archidiacre d'Arles, maître Guillaume sacriste de Riez, Amiel Botella sacriste d'Aix, Elzéar de Sabran, Raymond de Puyricard, Raynaud Porcelet de Sénas, Jean de Burlats le vieux, Philippe de Laveno.

22. GCNN, Arles, n 1359, 7 septembre 1295.

23. L Boniface VIII, $\mathrm{n}^{\circ} 1691,5$ février 1297.

24. LCu Boniface VIII, n 856, 2 janvier 1296.

25. En revanche, on connaît peu de choses sur l'entourage d'André: Guillaume de Rabastens est son procureur et clavaire en 1303 (ALBANÈS, Joseph Hyacinthe - Gallia christiana novissima, Avignon / par feu le chanoine J.-H. Albanès [...]; complétée, annotée et publiée par le chanoine Ulysse Chevalier [...]. Valence: impr. Valentinoise, 1920 [désormais GCNN, Avignon], $\mathrm{n}^{\circ}$ 783, 17 avril 1303).

26. GCNN, Avignon, $\mathrm{n}^{\circ} 755$.

27. GCNN, Avignon, $\mathrm{n}^{\circ}$ 758-759: il ne peut ainsi assister à l'élection de l'abbé de Saint-André de Villeneuve-lès-Avignon, Bertrand de Laudun, vers le début du mois de janvier 1291.

28. RYMER, Thomas - Fœdera, conventiones, literce et cujuscumque generis acta publica, inter reges Anglice et alios. T. 1-2. Hagae comitis (apud Joannem Neauline), 1745, pp. 77-78.

29. THEIS, Valérie - Le gouvernement pontifical du Comtat Venaissin (v. 1270-v. 1350). Rome: École Française de Rome, 2012, p. 230.

30. $R C A$, t. $44, \mathrm{n}^{\circ} 572$, pp. 757-758, 21 mai 1292, lettre royale aux officiers d'Avignon et de Tarascon pour faciliter à l'évêque le recouvrement des dettes auprès de particuliers.

31. GUENÉE, Benard - Entre l'Église et l'État. Quatre vies de prélats français à la fin du Moyen Âge (XIII XVe siècles). Paris: Gallimard, 1987. 
32. MOREMBERT, Henri Tribout de - "Ferrières, Pierre de“. in BAUDRILLART, Alfred; VOGT, Albert; ROUZIÈS, Urbain (dir.) - Dictionnaire d'histoire et de géographie ecclésiastique. T. 16. Paris: Letouzey et Ané, 1967, col.1287-1288; GIORDANENGO, Gérard - Lexikon des Mittelalters. T. 4. Erzkanzler bis Hiddensee. München. Zürich: Artemis, 1989, pp.397-398. Il est l'auteur d'un petit traité, De mero et mixto imperio (Naples, Bibl. Nazionale, III A 6, fol. 167av-168b, cité par Gérard GIORDANENGO, Lexikon des Mittelalters ...), d'additions à la Glose d'Accurse, au Digeste et au Code. On connaît le contenu de sa bibliothèque: POMMEROL, Marie-Henriette Jullien de; MONFRIN, Jacques (éd.) - Bibliothèques ecclésiastiques au temps de la papauté d'Avignon. T. 2. Inventaires de prélats et de clercs français. Édition. Paris: CNRS éditions, 2001, 2, 307.8, pp. 52-54.

33. Il est familier de Louis d'Anjou et l'assiste lors de sa mort en août 1297. BUGHETTI, P. Benvenutus (éd.) - Processus canonizationis et legendoe varice sancti Ludovici O. F. M. episcopi Tolosani, Analecta franciscana. T. 7. Quaracchi - Firenze: ex Typographia Collegii S. Bonaventurae, 1951, p. 76 et $\mathrm{n}^{\circ}$ 29, pp.452-455. PÉCOUT, Thierry - "Jacques Duèze, évêque de Fréjus (1300-1310)". Cahiers de Fanjeaux: Jacques Duèze-Jean XXII et le Midi 45 (2013), pp. 41-68.

34. Sur ce milieu: BOYER, Jean-Paul - "Le droit civil entre studium et cour de Naples". in BOYER, Jean-Paul; MAILLOUX, Anne; VERDON, Laure (dir.) - La justice temporelle dans les territoires angevins aux XIII et XIV e siècles. Théories et pratiques, colloque d'Aix-en-Provence, 21-23 février 2002. Paris: École française de Rome, 2005, pp. 47-82.

35. Sa première mention comme chancelier date du 15 avril 1296 (PALMIERI, Stefano - La cancelleria..., pp. 167 et n. 299); de même, 3 octobre 1298 (L Boniface VIII nº 2789). Bien que l'époque voie la charge de chancelier perdre de sa valeur au profit du lieutenant du logothète, l'activité de Pierre de Ferrières en Provence est loin d'être négligeable (Palmieri, Stefano - La cancelleria..., pp. 167-168). Sur les chanceliers et vice-chanceliers de Charles II: KIESEWETTER, Andreas - "La cancelleria angioina" ..., pp. 383-384: Pierre de Ferrières succède à Adam de Douzy, attesté en 1291 et mort le 25 août 1294 (Adam de Douzy, chanoine de Chartres, est élu archevêque de Cosenza mais fait reporter plusieurs fois le délai de sa consécration: L Nicolas IV, $\mathrm{n}^{\circ} 5481,29$ juin 1291); il est suivi lui-même par Jacques Duèze en 1308-1310. Digne-les-Bains, Arch. départementales des Alpes-de-Haute-Provence, 1G 25, 14 avril 1298.

36. L Boniface VIII, $\mathrm{n}^{\circ} 4080$.

37. Le 23 décembre 1299, il est nommé évêque de Lectoure, grâce à l'intervention de Boniface VIII dans l'élection du chapitre (L Boniface VIII, $\mathrm{n}^{\circ} 3289$ ), et en février suivant il réorganise le temporel suite aux aliénations de son prédécesseur (L Boniface VIII, $\mathrm{n}^{\circ}$ 3439). Le 22 décembre 1301, il est transféré à Noyon (L Boniface VIII, $\mathrm{n}^{\circ} 4260$ ).

38. L Boniface VIII, $\mathrm{n}^{\circ} 2790$, à la mort de l'archevêque de Salerno Filippo. Guillaume de Godonio est originaire du Quercy. Il est lieutenant du chancelier de Sicile en 1296 puis chancelier du duc de Calabre. Il obtient l'archevêché de Salerno après la renonciation de Pierre de Ferrières le 3 octobre 1298, puis reçoit plusieurs dispenses pour différer sa consécration entre 1299 et 1303 , grâce à l'appui de Robert de Calabre. Il est mort avant le 22 janvier 1306. PALMIERI, Stefano - La cancelleria..., pp. 169 et 176.

39. Pierre de Ferrières est élu archevêque par voie de scrutin dès le 23 août 1303, mais il n'est pourvu par le pape que le 30 janvier 1304: GCNN, Arles, n 1412. CADIER, Léon - Essai sur l'administration du royaume de Sicile ..., pp. 251-252. DOSSAT, Yves - "L'Université de Toulouse, Raymond VII, les Capitouls et le roi", pp. 75-76. GIORDANENGO, Gérard - "Ferrières, Pierre de". in ARABEYRE, Patrick; HALPÉRIN, Jean-Louis; KRYNEN, Jacques - Dictionnaire historique des juristes français, XII ${ }^{e} X^{e}$ siècle ..., pp. 327-328.

40. PAPON, Jean-Pierre - Histoire générale de Provence. T. 3. Paris: L'Imprimerie de Ph. D. Pierres, 1784, p. XLIX.

41. MONTI, Gennaro Maria - "Carlo II e i debiti angioino verso la Santa Sede". in MONTI, Gennario Maria - Da Carlo I a Roberto d'Angio. Ricerche e documenti. Trani: Vecchi, 1936, 
pp. 117-132 et ici pp. 121-123; HOUSLEY, Norman - The Italian Crusades. The Papal-Angevin Alliance and the Crusades against Christian Lay Powers, 1254-1343. Oxford: Claredon Press, 1982, p. 244.

42. PÉCOUT, Thierry - "Docteurs et professeurs de droit civil parmi les grands officiers de Provence angevine, 1246-1343". in MATHIEU, Isabelle; MATZ, Jean-Michel (dir.) - Formations et cultures des officiers et de l'entourage des princes dans les territoires angevins (milieu XIII ${ }^{e}-$ fin XV ${ }^{e}$ siècle) Percorsi di formazione e culture degli ufficiali e dell'entourage dei principi nei territori angioini (metà XIIIfine XV secolo) [en ligne]. Rome: Publications de l'École Française de Rome, 2019, pp. 119-142 [Consulté le 31 décembre 2019]. Accessible in https: //doi.org/10.4000/books.efr.4047.

43. La carrière de Guillaume de Mandagout est retracée par VIOLLET, Paul - "Guillaume de Mandagout, canoniste". in Histoire littéraire de la France. T. 34. Paris: Imprimerie Nationale, 1915, pp. 1-61, dont nous reprenons bon nombre de références.

44. GUILLEMAIN, Bernard - "Le milieu épiscopal et cardinalice de Bernard Gui". Cahiers de Fanjeaux: Bernard Gui et son monde 16 (1981), pp. 317-332, et ici pp. 324-325.

45. Son opuscule sur la date de Pâques composé après 1280 est destiné vraisemblablement au sénéchal de Beaucaire: BRUNEL, Clovis - "Une table pascale de Guillaume de Mandagout". Bibliothèque de l'École des chartes [en ligne] 84 (1923), pp. 161-165 [Consulté le 31 décembre 2015]. Accessible in https: //doi.org/10.3406/bec.1923.448690.

46. GCNN, Arles, $\mathrm{n}^{\circ} 2760,28$ août 1286.

47. L Boniface VIII, $\mathrm{n}^{\circ} 63$ et Gallia christiana, t. 3, Paris, 1725, Instr., $\mathrm{n}^{\circ}$ 5, col. 183-184: Guillaume est pourvu archevêque le 28 mars 1295, est consacré le 10 avril et reçoit le pallium le 13 avril suivant. L'archevêque délègue alors Bertrand Planterii, vicaire, et Rifridus Siguini, camérier, pour prendre possession de sa charge, ce qui est fait le $1^{\text {er }}$ mai. Il se rend à Embrun le 19 juin et fait son entrée dans la cité le 25 juin.

48. SCHULTE, Johann Friedrich von - Die Geschichte der Quellen und Literatur des Canonischen Rechts von Papst Gregor IX. bis zum Concil von Trient. Stuttgart: Ferdinand Enke, 1877, pp. 183-185. MOLLAT, Guillaume - "Guillaume de Mandagout". in NAZ, Raoul (dir.) - Dictionnaire de droit canonique. T. 5. Paris: Letouzey ey Ané, 1953, col.1077-1078. BÉGOU-DAVIA, Michèle - "Mandagout Guillaume de". in Arabeyre, Patrick; HALPÉRIN, Jean-Louis; KRYNEN, Jacques - Dictionnaire historique des juristes français, XII ${ }^{e}-X X^{e}$ siècle..., p. 533; BAGLIANI, Agostino Paravicini - Boniface VIII. Un pape hérétique? Paris: Editions Payot \& Rivages, 2003, p. 201. Le Tractatus de electionibus ou Libellus de episcoporum electionibus, composé de 60 chapitres et rédigé peu avant 1294, propose une synthèse de l'apport des décrétalistes plutôt qu'une réflexion théorique sur l'élection. Il s'agit d'un manuel de patricien (présentation analytique dans VIOLLET, Paul - "Guillaume de Mandagout, canoniste"..., pp. 1-61). VIOLLET, Paul - "Les élections ecclésiastiques au Moyen Âge d'après Guillaume de Mandagout". Revue canonique des églises 4 (1907), pp. 65-91; BALBERGHE, Émile van - "Une copie exceptionnelle du Libellus de episcoporum electionibus de Guillaume de Mandagout". in RAMAN, Anny; Manning, Eugéne (éd.) - Miscellanea Martin Wittek. Album de codicologie et de paléographie offert à Martin Wittek. Louvain-Paris: Peters, 1993, pp. 315-322; DÉLIVRÉ, Fabrice - "Le Libellus super electionibus de Guillaume de Mandagout (1286/1287). Histoire d'un succès dans l'Occident médiéval". in GOERING, Joseph Ward; DUSIL, Stephan; THEIR, Andreas (dir.) - Proceedings of the Fourteenth International Congress of Medieval Canon Law, Toronto, 5-11 August 2012. Cité du Vatican: Biblioteca Apostolica Vaticana, 2016, pp. 233-242; DÉLIVRÉ, Fabrice - "Mandagotus. Les avatars d'un traité juridique à succès". in BILOTTA, Maria Alesssandra (dir.) - Medieval Europe in Motion. Circulations juridiques et pratiques artistiques, intellectuelles et culturelles en Europe au Moyen Âge (XIII ${ }^{e}-X V^{e}$ siècle). Lisbonne, 25-25 février 2016, à paraître.

49. LC Clément $V$, $\mathrm{n}^{\text {os }} 7001,6865$.

50. L Boniface VIII, $\mathrm{n}^{\circ} 1919,1^{\mathrm{er}}$ juin 1297.

51. LCu Nicolas IV, $\mathrm{n}^{\text {os }} 6761,6763,6764,6774-6777,1^{\mathrm{er}}-21$ août 1291 , diverses instructions à Guillaume au sujet de sa mission auprès de Jacques, roi d'Aragon et sa citation à comparaître, et du clergé du royaume d'Aragon. LCu Nicolas IV, $\mathrm{n}^{\text {os }} 6762$ et $6835,1^{\text {er }}$ août et $1^{\text {er }}$ octobre 1291 , 
exhortations du pape au roi d'Aragon et appel à l'aide au roi de France. LCu Nicolas IV, $\mathrm{n}^{\circ} 6733$, 22 août 1291: lettre du pape au clergé de Majorque au sujet de l'envoi de Guillaume de Mandagout, son notaire, pour veiller à la prestation de fidélité au roi Jacques.

52. L Boniface VIII, $\mathrm{n}^{\circ} 827$ et App., $\mathrm{n}^{\text {os }} 5575$, 5577, 5579, 5580, 19 septembre 1295; L Boniface VIII, App., $n^{\circ} 5581$, après novembre 1295. LCu Boniface VIII, $\mathrm{n}^{\circ} 856,2$ janvier 1296 . L'accord débouche aussi sur la révocation de Frédéric III comme roi de Sicile et sur la renonciation par le roi d'Aragon ou sa mère Constance à toute aide en sa faveur. Il est probable alors que Guillaume de Mandagout ait eu à négocier avec Guillelm Llul, conseiller du roi d'Aragon, actif diplomate entre 1291 et 1301, et fréquemment en contact avec Charles II, mais leurs profils sociaux et intellectuels sont tout à fait différents: PÉQUIGNOT, Stéphane - Au nom du roi ..., pp. 240-241.

53. CHEVALIER, Ulysse - Regeste dauphinois. Répertoire chronologique et analytique des documents imprimés et manuscrits relatifs à l'histoire du Dauphiné, des origines chrétiennes à l'an 1349, Valence: L'Imprimerie Valentinoise, 1913-1926, ${ }^{\text {os }} 15863-15865$, 3 juillet 1301.

54. $L$ Benoît XI, $\mathrm{n}^{\circ} 847,19$ février 1304.

55. PÉCOUT, Thierry - Jacques Duèze..., pp. 41-68.

56. LCu Benoît XI, $\mathrm{n}^{\circ}$ 1121. LC Clément V, $\mathrm{n}^{\circ} 2228,23$ octobre 1307 (à propos de Carpentras); $\mathrm{n}^{\circ} 4775$, 11 octobre 1309. Son successeur Raymond Guilhem de Budos est nommé le 13 septembre 1310. THEIS, Valérie - Le gouvernement pontifical du Comtat Venaissin (v. 1270-v. 1350) ..., pp. 262-264 et 268-269.

57. AD13, B 1088 (48 folios), 25 octobre - $1^{\mathrm{er}}$ novembre 1304: procédure concernant la dévolution des biens de Roncelin de Lunel et de Montauban, qui teste en 1294. Les clauses de son testament n'ayant pas été respectées par son héritier Hugues Adémar, les biens passent donc au roi. Le 25 octobre 1304, les procureurs de Charles II, dont Pierre Gomberti, cherchent à éviter que les domaines de Montauban n'échoient au dauphin, avec la complicité des exécuteurs testamentaires, les évêques de Tricastin et de Vaison, le provincial et le custode OFM d'Avignon. Ces derniers s'en remettent à l'archevêque, qui préconise de vendre la baronnie au dauphin, ce qui suscite l'opposition de Charles II. Guillaume de Mandagout recule alors et proteste de son ignorance des clauses du testament, expédiant deux lettres aux deux évêques où il récuse son conseil. ALBANÈS, Joseph Hyacinthe - Histoire des évêques de Saint-Paul-Trois-Châteaux au quatorzième siècle. Corrections et documents. Montbéliard: Imprimerie de P. Hoffmann, 1885, pp. 3-5 et $\mathrm{n}^{\text {os }}$ 3-4. VIOLLET, Paul - “Guillaume de Mandagout, canoniste”..., pp. 1-61. PÉCOUT, Thierry (dir.); BERNARDI, Philippe; BONNAUD, Jean-Luc; CASSIOLI, Marco; MAILLOUX, Anne et al. (éd.) L'enquête générale de Leopardo da Foligno dans le comté de Forcalquier (juin-septembre 1332). Paris: Comité des travaux historiques et scientifiques, 2017, pp. 1007-1009.

58. THEIS, Valérie - Le gouvernement pontifical du Comtat Venaissin (v. 1270-v. 1350)..., pp. 263-264.

59. PÉCOUT, Thierry - "Aux origines d'une culture administrative: le clergé des cathédrales et la genèse d'une comptabilité princière en Provence à la fin du XIII ${ }^{\mathrm{e}}$ siècle”. in PÉCOUT, Thierry (dir.) - De l'autel à l'écritoire. Aux origines des comptabilités princières en Occident (XII ${ }^{e}-X{ }^{e}$ siècle), Actes du colloque international d'Aix-en-Provence, 13-14 juin 2013. Paris: De Boccard, 2017, pp. 49-67.

60. TOOMASPOEG, Kristjan - Decimae. Il sostegno economico dei sovrani alla Chiesa del Mezzogiorno nel XIII secolo. Dai lasciti di Eduard Sthamer e Norbert Kamp. Rome: Roma Viella, 2009, pp. 71-83; TOOMASPOEG, Kristjan - "L'Église et la fiscalité au royaume de Sicile (XI ${ }^{\text {e-XIV }}{ }^{\mathrm{e}}$ siècles)". in MENJOT, Denis; SÁNCHEZ MARTÍNEZ, Manuel (dir.) - El dinero de Dios. Iglesia y fiscalidad en el Occidente medieval (siglos XIII-XV). Madrid: Instituto de Estudios Fiscales, 2011, pp. 91-100. On dispose désormais de précieux travaux sur la collecte de la décime et le milieu des collecteurs, avec une attention particulière pour les diocèses de Provence: LE ROUX, Amandine - "Mise en place des collecteurs et des collectories dans le royaume de France et en Provence (1316-1378)". Lusitania sacra. 0 papado de Avinhão nos reinos do Ocidente 22 (2010), pp. 45-62; LE ROUX, Amandine - "Des collecteurs spécialisés aux collecteurs généralistes, l'établissement du système collectoral en Provence (1249-1514)". in MORELLO BAGET, Jordi (dir.) - Financiar el reino terrenal. La 
contribución de la Iglesia a finales de la Edad Media (s. XIII-XVI). Barcelone: CSIC, 2013, pp. 107-129; LE ROUX, Amandine - "L'écrit, un outil de gouvernement financier de la papauté. L'exemple de la correspondance entre la papauté et ses collecteurs". in BÉRENGER, Agnès; DARD, Olivier (éd.) Gouverner par les lettres, de l'Antiquité à l'époque contemporaine. Actes du colloque de Metz, 10-12 octobre 2013. Metz: Centre de Recherche Universitaire Lorrain d'Histoire, 2015, pp. 287-311; LE ROUX, Amandine - "Le recouvrement de la décime par les collecteurs pontificaux de 1316 à 1503 (royaume de France et Provence)". in BALOUP, Daniel; SÁNCHEZ MARTíNEZ, Manuel (dir.) - Partir en croisade à la fin du Moyen Âge. Financement et logistique. Toulouse: Presses Universitaires du Midi, 2015, pp.55-80; LE ROUX, Amandine - "Pratiques comptables du gouvernement pontifical. L'histoire scripturale des comptes des collecteurs pontificaux provençaux (1274-1406)". Comptabilités [en ligne] 10 (2019) [Consulté le 31 décembre 2019]. Accessible in https:// journals.openedition.org/comptabilites/2545.

61. GCNN, Marseille, $\mathrm{n}^{\circ} 322,21$ mars 1287, à Rome. Quand Nicolas IV le pourvoit du siège de Marseille le 17 avril 1289, c'est le cardinal de Porto Bernard qui est chargé de le consacrer (GCNN, Marseille, $\mathrm{n}^{\circ} 323$ et L Nicolas IV, $\mathrm{n}^{\circ}$ 943; BOESPFLUG, Thérese - La Curie au temps de Boniface VIII. Étude prosopographique. Rome: Istituto Storico Italiano per il Medioevo, 2005, $\mathrm{n}^{\circ}$ 204, p. 125).

62. L Nicolas IV, 15 juillet 1290. En 1296, il assiste ainsi Boniface VIII pour régler le délicat conflit entre Étienne de Monte Areno, abbé de Montmajour, et Aimone di Montanaro (ou Aymon de Montagne), grand maître de Saint Antoine. Ce différend finit par mettre en jeu les intérêts politiques du dauphin face à ceux de Charles II. Le conflit porte notamment sur la gestion des legs et des testaments disputés entre l'hôpital et le prieuré OSB de Saint-Antoine. L Boniface VIII, $\mathrm{n}^{\circ} 2032$ et MISCHLEWSKI, Adalbert - Un ordre hospitalier au Moyen Âge. Les chanoines réguliers de Saint-Antoine-en-Viennois. Grenoble: Presses Universitaires de Grenoble, 1995, pp. 27-37 et n ${ }^{\circ}$, pp. 146-155, 10 juin 1297, aux côtés de l'archevêque d'Embrun et de l'évêque de Viviers, Guillaume OFM. L'abbé de Montmajour s'efforce en effet de reprendre le contrôle de l'hôpital Saint-Antoine, qui relève en théorie de sa mense, fort de la décision de Clément IV qui attribuait en juillet 1267 les bénéfices et dons indéterminés en priorité à son prieuré détenteur des reliques, au détriment de l'hôpital. Malgré un premier arbitrage de Bertrand d'Anguissola, évêque de Nîmes, l'affaire est portée devant Nicolas IV qui délègue une commission de cardinaux, avec Bernard d'Anguissola, Giacomo Colonna, Pietro Peregrosso et Benedetto Caetani. Le conflit devenant particulièrement violent et embrasant la région, il met en jeu le dauphin protecteur des Antonins et Charles II qui obtient la tutelle du prieuré. L'arbitrage finalement conclu à Romans en 1292 est contesté par Montmajour qui en appelle à Boniface VIII. Le pape désigne alors Durand de Tres Eminas en 1296, mais la procédure ne connaît une conclusion qu'en juin 1297 du fait des occupations fiscales de l'évêque de Marseille. Le pape prononce ainsi le 10 juin 1297 une séparation entre les Antonins érigés en ordre canonial et l'abbaye de Montmajour qui reçoit une compensation financière.

63. FAILLON, M. - Monuments inédits sur l'apostolat de Sainte Marie Madeleine en Provence. T. 2. Paris: Jacques-Paul Migne, 1848, $\mathrm{n}^{\circ}$ 98, col.841-846; SAXER, Victor - "Les ossements dits de sainte Marie-Madeleine conservés à Saint-Maximin-la-Sainte-Baume”. Provence historique 27 (1977), pp. 257-302; COULET, Noël - "Un couvent royal: les Dominicaines de Notre-Dame-de-Nazareth d'Aix au XIII e s.". Cahiers de Fanjeaux: Les Mendiants en Pays d'Oc au XIII e siècle 8 (1973), pp. 233-262.

64. L Boniface VIII, $\mathrm{n}^{\circ} 1818,18$ février 1297 , faculté d'absoudre les censures contre ceux qui n'auraient pas payé correctement la décime; $n^{\circ} 3064,28$ mai 1299, n 3467, 3469, 17-20 février $1300, n^{\circ} 3641,21$ juin $1300, n^{\circ} 4173,28$ septembre 1301, ordres d'assignation aux Spini et aux divers mercatores camere; $\mathrm{n}^{\circ}$ 3068, 18 juin 1299, sur l'exemption de décime en faveur des moniales de Notre-Dame de Nazareth d'Aix, à la demande de Charles II. Assignations du produit de la décime à la chambre royale, en vue d'armer des navires, le 8 juillet 1298 (AD13, 5G 17, $\mathrm{n}^{\circ} 114$ ). LCu Boniface VIII, $\mathrm{n}^{\circ}$ 4396-4397, les 8 et 24 septembre 1301. GCNN, Marseille, $\mathrm{n}^{\circ} 343,7$ avril 1296: l'Hôpital n'est pas tenu à la décime comme l'a rappelé le pape; BLANCARD, Louis - Documents 
inédits sur le commerce de Marseille au Moyen Âge. T. 2-4. Pièces commerciales diverses tirées des archives marseillaises du XIII ${ }^{e}$ siècle. Marseille: Typographie et Litographie Barlatier-Feissat Père et Fils, $1885, \mathrm{n}^{\circ}$ 91, pp. 450-451, quittance du 21 août 1296 pour la décime du diocèse de Marseille levée par le sacriste Guillaume de Florensiaco, pour l'an 1, par des marchands toscans; GCNN, Marseille, $\mathrm{n}^{\circ} 355,18$ février 1297; $\mathrm{n}^{\circ} 361,1^{\mathrm{er}}$ octobre 1298; $\mathrm{n}^{\circ} 358,17$ mai 1298: lettre du collecteur au sacriste Guillaume de Florensiaco sur une sentence d'excommunication contre l'aumônier de Saint-Victor; $\mathrm{n}^{\circ}$ 360, 8 juillet 1298: quittance de Charles II après versement du produit de la décime par des sociétés florentines; $\mathrm{n}^{\circ} 364$ (et BLANCARD, Louis - Documents inédits sur le commerce de Marseille au Moyen Âge..., $\mathrm{n}^{\circ}$ 112), 14 juillet 1299: instructions du pape à propos des assignations aux sociétés; BLANCARD, Louis - Documents inéditssur le commerce de Marseille au Moyen Âge..., $\mathrm{n}^{\circ}$ 112, quittance par les Spini au collecteur Guillaume de Florensiaco et opérations de change entre royaux et provençaux coronats, 16 juillet 1299; GCNN, Marseille, $\mathrm{n}^{\circ}$ 368, 24 septembre 1302: lettre du pape à Durand; $n^{\circ} 372,13$ janvier 1304, lettre de Benoît XI. L Benoît XI, $\mathrm{n}^{\circ} 181$, 13 janvier 1304, assignations aux Ciruli de Florence. L Boniface VIII, $\mathrm{n}^{\circ} 497^{\mathrm{b}}, 28$ octobre 1295, concession de la décime au roi de Sicile; L Boniface VIII, $\mathrm{n}^{\circ} 1818,18$ février 1297, pouvoir de lever l'excommunication pour défaut de paiement; LCu Boniface VIII, $\mathrm{n}^{\circ} 2888,1^{\mathrm{er}}$ octobre 1298; L Boniface VIII, $\mathrm{n}^{\circ}$ 3064, 20 mai 1299; L Boniface VIII, $\mathrm{n}^{\circ}$ 3064, 28 mai 1299 et $\mathrm{n}^{\circ}$ 3467, 17 février 1300, assignations aux Spini; L Boniface VIII, $n^{\circ} 3495,4$ janvier 1300; L Boniface VIII, $n^{\circ} 3641,21$ juin 1300; L Boniface VIII, $\mathrm{n}^{\circ}$ 4173, 28 septembre 1301; LCu Boniface VIII, $\mathrm{n}^{\circ}$ 4396-4397, 8 et 24 septembre 1301. En 1296, sur le conflit opposant les Mozzi, Spini et Clarenti au sénéchal, à propos des reversements non effectués, ce qui conduit ce dernier à saisir leurs marchandises dans le port de Marseille: HOUSLEY, Nornam - The Italian Crusades..., pp. 236. ASV, Instr. Misc. 359: l'instrument est passé dans le palais épiscopal le 23 août 1303, en présence d'Hugues Lautandi vicaire du Castellet, du prêtre Pierre Picordi de Méounes, de Bernard de Hamis, Pons Samuelis, du diocèse de Nîmes, de Pons de Crota et de son fils, de Beaucaire. Il s'agit vraisemblablement de membres de la familia de l'évêque Durand. Le procureur de la société des Spini, à l'appui de sa procuration du 5 décembre 1301, reconnaît avoir perçu divers montants de la part des sous-collecteurs, à la date du 24 juin 1303, échéance de la deuxième année, y compris les reliquats de la première année de la décime concédée par le pape en 1301. Les sous-collecteurs sont alors, pour Gap: Hugues de Sancto Marcello, chanoine, et Henri Edulphi, précenteur; pour Sisteron, le chanoine Raymond de Mura; pour Apt, le sacriste Pierre Ruffi; pour Cavaillon, le chanoine Bertrand Gerardi; pour Arles, le prévôt Bertrand et l'archiprêtre Bertrand d'Eyguières; pour Avignon, le chanoine Bertrand deAuriaco; pour Aix, le chanoine Visdomino dei Visdomini; pour Toulon, le chanoine Marquesius d'Anduze; pour Fréjus, l'archidiacre Boniface Dalmacii; pour Riez, l'archidiacre Raymond Robaudi; pour Carpentras, le chanoine Siffred Raffini; pour Saint-Paul-Trois-Châteaux, le sacriste Pons Salardi; pour Orange, le sacriste Hugues Jordanis; pour Vaison, le prévôt Guillaume de Seinhano; pour Marseille, le chanoine Raymond Egidii..

65. LCu Boniface VIII, $n^{\circ} 1637,1^{\text {er }}$ août 1296, et L Boniface VIII, App., $n^{\circ} 5591$, août 1296, sommes allouées à la construction de galères; L Boniface VIII, registre caméral, $\mathrm{n}^{\text {os }} 5468-5469,13$ décembre 1296, assignations aux sociétés florentines de sommes destinées à la construction de galères; L Boniface VIII, registre caméral, $\mathrm{n}^{\circ}$ 5500, 7 février 1298, financement d'armement naval. Sur les constructions navales à Marseille à ce moment et le rôle de Durand: KIESEWETTER, Andreas "Karl II. von Anjou, Marseille und Neapel". in BONNOT-RAMBAUD, Isabelle (dir.) - Marseille et ses rois de Naples. La diagonale angevine (1265-1382). Marseille / Aix-en-Provence: Archives Municipales / Edisud, 1988, pp. 61-75, et ici pp. 64-65; BRESC, Henri - "Marseille dans la guerre des Vêpres siciliennes". in BONNOT-RAMBAUD, Isabelle (dir.) - Marseille et ses rois de Naples..., 1988, pp. 43-49. 66. PÉCOUT, Thierry - "Les évêques de Gap, XII ${ }^{\mathrm{e}}-\mathrm{XIV}$ e siècle: les enjeux d'un contrôle politique entre Provence et Dauphiné”. in PLAYOUST, Pierre-Yves (dir.) - Gap et les territoires gapençais de la préhistoire à nos jours. Grenoble: Presses universitaires de Grenoble, 2016, pp. 15-53. 
67. Plus largement, sur les premières étapes d'un processus menant aux assemblées d'états: HÉBERT, Michel - "Une identité mise en scène: les premières assemblées représentatives dans les comtés de Provence et de Forcalquier (XIII ${ }^{\mathrm{e}}$-début XIV ${ }^{\mathrm{e}}$ siècles)". in BOYER, Jean-Paul; MAILLOUX, Anne; VERDON, Laure (dir.) - Identités angevines. Entre Provence et Naples (Aix-en-Provence, 20-22 octobre 2011). Aix-en-Provence: Presses universitaires de Provence, 2016, pp. 183-194.

68. BOYER, Jean-Paul - "Sacre et théocratie. Le cas des rois de Sicile Charles II (1289) et Robert (1309)". Revue des Sciences philosophiques et théologiques 81/4 (1997), pp. 561-607.

\section{RÉSUMÉS}

L'épiscopat et les prévôts des cathédrales ont joué sous Charles II un rôle politique accru, s'intégrant aux rouages du gouvernement sous une forme plus aboutie au regard du règne de Charles d'Anjou. La diplomatie en constitue l'un des aspects, quand le magistère de la parole, les compétences en matière de droit canonique et les réseaux personnels ou bénéficiaux, sont mis à profit pour servir les ambitions méditerranéennes de la monarchie.

In comparison to the reign of Charles of Anjou, the bishops and provosts of the cathedrals of Provence gained an increasing political importance under Charles II inasmuch as they were able to smoothly integrate themselves into the government apparatus. One aspect of this integration is diplomacy, which through the magisterium of speech, skills in canon law and personal or beneficial networks serves the Mediterranean ambitions of the Monarchy.

\section{INDEX}

Keywords : Épiscopat, Provence, Diplomatie, Prévôt, Angevins de Provence-Sicile

\section{AUTEUR \\ THIERRY PÉCOUT}

Université de Saint-Étienne, UMR LEM-CERCOR 42023 Saint-Étienne Cedex 2, France. thierry.pecout@univ-st-etienne.fr. https://orcid.org/0000-0001-9969-3334. 